\title{
Low-rate accretion onto isolated stellar-mass black holes
}

\author{
G. M. Beskin ${ }^{1,2}$ and S. V. Karpov ${ }^{1,2}$ \\ 1 Special Astrophysical Observatory, Nizhnij Arkhyz, Karachaevo-Cherkesia 369167, Russia \\ e-mail: beskin@sao.ru \\ 2 Isaac Newton Institute of Chile, SAO Branch, Russia
}

Received 1 April 2004 / Accepted 28 April 2005

\begin{abstract}
Magnetic field behaviour in a spherically-symmetric accretion flow for parameters typical of single black holes in the Galaxy is discussed. It is shown that in the majority of Galaxy volume, accretion onto single stellar-mass black holes will be spherical and have a low accretion rate $\left(10^{-6}-10^{-9}\right.$ of the Eddington rate). An analysis of plasma internal energy growth during the infall is performed. Adiabatic heating of collisionless accretion flow due to magnetic adiabatic invariant conservation is $25 \%$ more efficient than in the standard non-magnetized gas case. It is shown that magnetic field line reconnections in discrete current sheets lead to significant nonthermal electron component formation. In a framework of quasi-diffusion acceleration, the "energy-radius" electron distribution is computed and the function describing the shape of synchrotron radiation spectrum is constructed. It is shown that nonthermal electron emission leads to formation of a hard (UV, X-ray, up to gamma), highly variable spectral component in addition to the standard synchrotron optical component first derived by Shvartsman generated by thermal electrons in the magnetic field of accretion flow. For typical interstellar medium parameters, a black hole at $100 \mathrm{pc}$ distance will be a $16-25^{\mathrm{m}}$ optical source coinciding with the highly variable bright X-ray counterpart, while the variable component of optical emission will be about $18-27^{\mathrm{m}}$. The typical time scale of the variability is $10^{-4} \mathrm{~s}$, with relative flare amplitudes of $0.2-6 \%$ in various spectral bands. Possible applications of these results to the problem of search for single black holes are discussed.
\end{abstract}

Key words. accretion, accretion disks - magnetic fields

\section{Introduction}

Even though more than 60 years have passed since the theoretical prediction of black holes as an astrophysical objects (Oppenheimer \& Snyder 1939) in some sense they have not been discovered yet. To identify an object as a black hole, one needs to show that its mass exceeds $3 M_{\odot}$, its size is close to $r_{\mathrm{g}}=2 G M / \mathrm{c}^{2}$ and it has an event horizon instead of a normal surface - the distinguishing property of black holes which separates them from massive compact objects of finite size in some theories of gravity (Will 1998). However, only the two former criteria are used now for selection of black hole candidates of two types: a) with masses of 5-18 $M_{\odot}$, in X-ray binaries (see, for example, Greiner et al. 2001); and b) supermassive black holes in galaxy nuclei with masses of $10^{6}-10^{10} M_{\odot}$ (Shields 1999). Existence of the event horizon in such objects is usually implied by the absence of periodic pulsations of the $\mathrm{X}$-ray emission from strong regular magnetic fields (the black hole "no-hair" theorem) and I type X-ray flares due to thermonuclear bursts of the accreted matter on the surface of the neutron star. At the same time, typical masses of X-ray pulsars and bursters are close to the typical neutron star value of $1.4 M_{\odot}$ while black hole candidates, missing pulsations and X-ray flares, have masses of 5-18 $M_{\odot}$ (Miller et al. 1998). The absence of an event horizon in low-mass objects is not a proof of its existence in higher-mass ones.

High accretion rates in X-ray binaries and active galactic nuclei result in the screening of regions close to the event horizon, and the most luminous parts of accretion flow are situated at distances of 10-100 $r_{\mathrm{g}}$ (Chakrabarti 1996; Cherepashchuk 2003) where general relativity effects are negligible.

There is a very effective way to get information about the innermost parts of accretion disks in X-ray binaries as well as AGNs - the investigation of the broad (and sharp) iron $\mathrm{K}_{\alpha}$ fluorescent emission line (see review by Reynolds \& Nowak 2003). Its intensity and shape depend on the accreted plasma distribution and behaviour until the last stable orbit $\left(0.62 r_{\mathrm{g}}\right.$ for a extremely spinning Kerr black hole and $3 r_{\mathrm{g}}$ for a Schwarzschild one) (Miller et al. 2004; Miniutti et al. 2004). However since the photons generated at the different distances from the horizon are mixed in the line profile it is not possible to extract the manifestations of gravitational fields close to the horizon only. This may be possible by study of variability of the iron line (Reynolds et al. 2004).

At the same time, single stellar-mass black holes, which accrete interstellar medium of low density $\left(10^{-2}-1 \mathrm{~cm}^{-3}\right)$, are the ideal case for detection and study of the event horizon. Shvartsman (1971) first demonstrated that an emitting halo of accreted matter forms around such objects and generates 
optical featureless emission. The majority of such emission comes from the regions near the horizon at (3-5) $r_{\mathrm{g}}$. Spherical accretion onto the single stellar-mass black holes has been studied in detail in the works of several authors (Bisnovatyi-Kogan \& Ruzmaikin 1974; Meszaros 1975; Ipser \& Price 1977, 1982) and the main conclusions of Shvartsman have been confirmed.

The most striking property of the accretion flow onto the single black hole is its inhomogeneity - the clots of plasma act as a probe testing the space-time properties near the horizon. The characteristic timescale of emission variability is $\tau_{\mathrm{v}} \sim r_{\mathrm{g}} / c \sim 10^{-4}-10^{-5} \mathrm{~s}$ and such short stochastic variability may be considered as a distinctive property of black hole as the smallest possible physical object with a given mass. Its parameters - spectra, energy distribution and light curves - carry important information on space-time properties of the horizon (Beskin \& Shvartsman 1976).

The general observational appearance of a single stellarmass black hole at typical interstellar medium densities is the same as other optical objects without spectral lines DC-dwarfs and ROCOSes (Radio Objects with Continuous Optical Spectra, a subclass of blazars) (Beskin \& Mitronova 1991; Pustilnik 1977; Shvartsman 1977; Beskin et al. 2000). The suggestion that isolated BHs can be among them is the basis of the observational programme of search for isolated stellar-mass black holes - MANIA (Multichannel Analysis of Nanosecond Intensity Alterations). It uses photometric observations of candidate objects with high time resolution, special hardware and data analysis methods (Shvartsman 1977; Beskin et al. 1997).

In observations using the 6-m telescope of the Special Astrophysical Observatory of 40 DC-dwarfs and ROCOSes, only upper limits for variability levels of $20 \%-5 \%$ on the timescales of $10^{-6}-10 \mathrm{~s}$, respectively, were obtained, i.e. BHs were not detected (Shvartsman et al. 1989a,b; Beskin et al. 2000).

Recently, some evidences appeared that single stellar-mass black holes may be found among the stationary unidentified gamma-ray sources (Gehrels et al. 2002), gravitational lenses causing long-lasting MACHO events (Bennett et al. 2001) and white dwarf - black hole binaries detected by means of selfmicrolensing flashes (Beskin \& Tuntsov 2002). In the last case, the mass transfer from the white dwarf is absent and a black hole behaves as a single one.

In the present work we study spherical accretion onto a single stellar-mass black hole at low accretion rates of $10^{8}-10^{13} \mathrm{~g} / \mathrm{s}$. This corresponds to the range of interstellar medium densities of $0.002-0.1 \mathrm{~cm}^{-3}$ and a $10 M_{\odot}$ object moving with a velocity of $20-40 \mathrm{~km} \mathrm{~s}^{-1}$ (Bondi \& Hoyle 1944). This is true for about $90 \%$ of the Galaxy volume (McKee \& Ostriker 1977).

Spherical accretion with equipartition of energies, i.e. with roughly equal densities of the magnetic and kinetic energies of plasma has been considered in many papers (Shvartsman 1971; Bisnovatyi-Kogan \& Ruzmaikin 1974; Kowalenko \& Melia 1999; Ipser \& Price 1977, 1982). The uniqueness of our approach is in taking into account the significantly non-thermal nature of electron energy distribution function (its synchrotron emission determines the appearance of a black hole). It may be roughly considered as a superposition of two components (this approach is known as "hybrid plasma", see Coppi (1999) and references therein) - thermal electrons and accelerated electron beams, formed in current sheets where magnetic energy is dissipated in a way similar to solar flares (Pustilnik 1978, 1997). The latter process supports the equipartition of energies. As a result, the emission of the accretion flow consists of a quasistationary "thermal" part with a wide-band spectrum from infrared to ultraviolet, and a highly variable flaring nonthermal component. Each such flare is generated due to the motion of the accelerated electron beam in the magnetic field. Its light curve carries information on the magnetic and gravitational field structure near the black hole horizon. Nonthermal luminosity reaches several percents of the total luminosity and may even exceeds it at low rates, while its spectrum covers spectral bands from optical to hard X-ray. This result leads to possible modifications of the search strategy.

In Sect. 2, the main characteristics of accretion onto single black holes in the Galaxy are discussed.

In Sect. 3, the electron distribution function in phase space is built, in Sect. 4, the thermal and nonthermal component luminosities are determined, and in Sect. 5 the shape of its spectra is studied.

In Sect. 6, the temporal behaviour of single electron beam emission is studied and some conclusions on the variability of accretion flow are made.

In Sect. 7, the main results of this work are summarized, and in Sect. 9, possible directions of future work are discussed.

\section{Nature of accretion and basic parameters of the model}

\subsection{Accretion rate for different parameters of black holes and interstellar medium}

Contemporary models of massive star evolution, its dynamics and data on black hole candidates in X-ray binaries and microlensing events suggest that the most probable mass $M$ of a single black hole is $10 M_{\odot}$ (Greiner et al. 2001; Fryer \& Kalogera 2001; Agol \& Kamionkowski 2002), while its velocity is in the $10-50 \mathrm{~km} \mathrm{~s}^{-1}$ range and the gas capture crosssection is defined by the Bondi radius $r_{\mathrm{c}}$ (Bondi \& Hoyle 1944):

$r_{\mathrm{c}}=\frac{2 G M}{V^{2}+c_{\mathrm{s}}^{2}}$

where $c_{\mathrm{s}}$ is the sound speed in the interstellar medium.

The interstellar medium consists of at least three components (McKee \& Ostriker 1977) - cold $\left(T \sim 10^{2} \mathrm{~K}\right)$ and dense $\left(n \sim 10^{2} \mathrm{~cm}^{-3}\right)$ neutral hydrogen clouds, warm partly ionized hydrogen $\left(n \sim 0.1-0.2 \mathrm{~cm}^{-3}, T \sim 10^{4} \mathrm{~K}\right)$ ones and fully ionized coronal hydrogen $\left(n \sim 0.002 \mathrm{~cm}^{-3}, T \sim 10^{6} \mathrm{~K}\right)$ clouds. Motion may be subsonic as well as supersonic - the sound speed changes in the $1.5-150 \mathrm{~km} \mathrm{~s}^{-1}$ range.

For the black hole moving supersonically in a uniform medium, an accretion rate is determined by the behaviour of matter behind the gravitation centre, where the tangential gas velocity component vanishes in the shock wave and the gas 


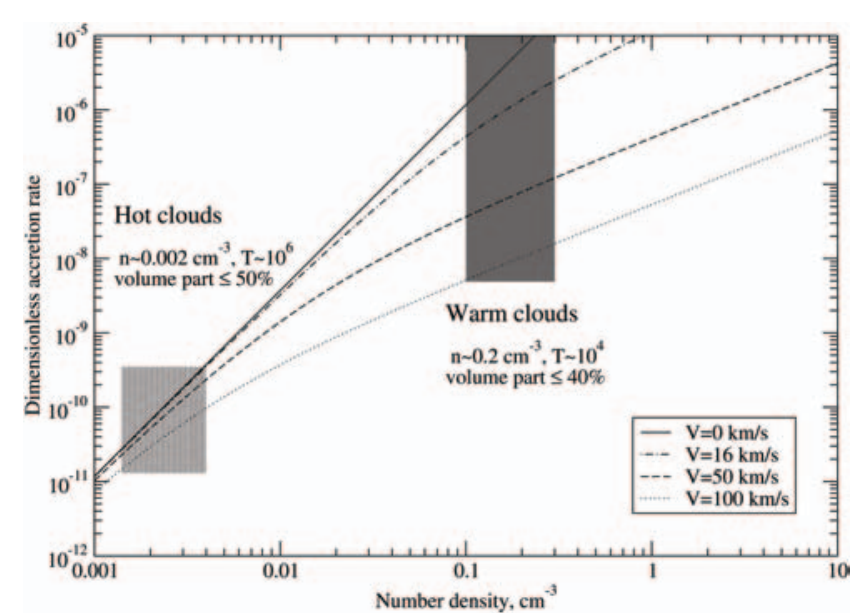

Fig. 1. Dimensionless accretion rates $\dot{m}=\dot{M} c^{2} / L_{\text {edd }}$ as a function of interstellar medium density for various black hole velocities.

falls towards the black hole in a wide cone (Bondi \& Hoyle 1944; Shvartsman 1971; Illarionov \& Sunyaev 1975; Font \& Ibanez 1998). For the typical case of interstellar medium that is collisionless on scales of the capture radius, a mode of accretion from a very thin "tail", where the tangential velocity vanishes due to the magnetic field, is possible. The thickness of such a tail may be estimated as

$\frac{r_{\text {tail }}}{r_{\mathrm{c}}} \approx\left(\frac{B_{\infty}^{2} / 8 \pi}{\rho V^{2} / 2}\right)^{1 / 2} \approx \frac{c_{\mathrm{s}}}{V}$,

where $B_{\infty}$ is the magnetic field strength at infinity, $V$ is the black hole velocity and rough equipartition of thermal and magnetic energies in the interstellar medium is taken into account. Accretion flow is continuous due to interstellar magnetic fields - the proton Larmor radius is $10^{7}-10^{8} \mathrm{~cm}$ which is at least $10^{6}$ times smaller than $r_{\mathrm{c}}$. The interstellar magnetic field in the Galaxy $\left(10^{-4}-10^{-5} \mathrm{G}\right)$ is frozen-in. The accretion rate for such a regime is determined by the expression (Bondi \& Hoyle 1944)

$\dot{M}=\frac{4 \pi G^{2} M^{2} \rho}{\left(V^{2}+c_{\mathrm{s}}^{2}\right)^{3 / 2}}$,

where $\rho$ in the interstellar medium density.

Using the expression for the Eddington accretion rate $\dot{M}_{\text {edd }}=L_{\text {edd }} / c^{2}=\frac{2 \pi m_{\mathrm{p}} c r_{\mathrm{g}}}{\sigma_{\mathrm{T}}}=1.4 \times 10^{18}\left(M / 10 M_{\odot}\right) \mathrm{g} / \mathrm{s}$, where $m_{\mathrm{p}}$ is the proton mass, $\sigma_{\mathrm{T}}$ is the Tompson cross-section, we have a useful normalization, measuring the mass in units of $10 M_{\odot}$, density in $1 \mathrm{~cm}^{-3}$, and velocity in $16 \mathrm{~km} \mathrm{~s}^{-1}$

$\dot{m}=\dot{M} / \dot{M}_{\text {edd }}=1.3 \times 10^{-5} M_{10} n_{1}\left(V^{2}+c_{\mathrm{s}}^{2}\right)_{16}^{-3 / 2}$.

In Fig. 1, the dependence of $\dot{m}$ on $n$ is shown for various velocities of the black hole motion (the dependence of the sound speed $c_{\mathrm{s}}$ on the temperature $T$ due to hydrostatic equilibrium of the ISM is also taken into account). Only in the cold clouds the accretion rate may reach the Eddington level, but due to the relative rareness of such clouds (5\% of the Galactic volume) this case is improbable. Note however that a black hole may initially be born in such a cloud, and so become a very bright source with a luminosity up to $10^{38}-10^{40} \mathrm{erg} / \mathrm{s}$ (and so, be a socalled "ultraluminous" source (Roberts et al. 2002), like those observed in other galaxies). For the warm hydrogen, whose volume fraction $\approx 40 \%$, the accretion rate will be $10^{-6}-10^{-8}$, while for hot hydrogen $(\approx 50 \%$ volume) it will be significantly lower. Later, we will assume such cases as the most typical for single black holes in the Galaxy.

\subsection{The role of interstellar medium inhomogeneities. Spherical or disc-like accretion?}

The observational appearance of the accreting black holes depends crucially on the regime of matter flow near the relativistic object. So, in binaries the captured angular momentum of matter is high enough, and a disc-like accretion regime takes place (Shakura \& Sunyaev 1973). For the case of accretion from interstellar medium, however, this problem is more complicated.

In the recent series of papers on the analysis of accretion onto single black holes, it was reasoned that a disc-like regime is realized for such a case also (Fujita et al. 1998; Agol \& Kamionowski 2002; Chisholm et al 2003). However, it seems they used overestimated values for the captured angular momentum.

Davies \& Pringle (1980) consider analytically the problem of accretion onto the moving gravitational centre of nonuniform interstellar medium (inhomogeneities of density as well as of velocity are considered) and show that, in the first order of inhomogeneities, the magnitude accretion rate is still described by the Bondi-Hoyle formula (3), and the captured angular momentum is zero.

The latter result may easily be understood by considering a simple model of accretion from the tail in which the traversal velocity component vanishes. This tail, on which the angular momentum is zero by definition, will not, in general, be a straight line, and the capture cross-section will not be a circle, and so, the total captured angular momentum will become zero too. In a more realistic picture, however, the role of gas pressure is important, the matter accretes in a wide cone, and the total captured angular momentum may be significant, although, as it was shown by numerical simulation results of Sawada et al. (1989), Ruffert (1997, 1999), it is much smaller than the usually-used estimation of Illarionov \& Sunyaev (1975):

$l_{\mathrm{m}}=\frac{1}{4} \beta V_{\mathrm{K}} r_{\mathrm{c}}$,

(where $\beta=\frac{\Delta \rho}{\rho}, \frac{\Delta V}{V}-$ relative density and velocity variations on $2 r_{\mathrm{c}}$ scale, $V_{\mathrm{K}}=\sqrt{\frac{V^{2}+c_{\mathrm{s}}^{2}}{2}}-$ Keplerian speed at the capture radius). So, this estimation may be considered as an upper limit in a very narrow class of objects.

Detailed analysis and numerical simulations leads to the more realistic expression for the captured angular momentum (Ruffert 1997, 1999):

$l \sim 0.1 l_{\mathrm{m}}$.

For the spherical accretion regime the specific angular momentum of the captured matter must be smaller than that of the 
black hole last stable orbit, i.e. $l<\sqrt{3} c r_{\mathrm{g}}$ for Schwarzschild metric. Then

$\beta<40 \sqrt{6} \frac{\sqrt{V^{2}+c_{\mathrm{s}}^{2}}}{s}$.

Due to the characteristic dispersion scale in the interstellar medium being of the order of $(\Delta V)^{2}=1.1(r / 1 \mathrm{pc})^{0.76}\left(\mathrm{~km} \mathrm{~s}^{-1}\right)^{2}$ (Larson 1981; Falgarone \& Phillips 1990), then

$1.1\left(\frac{2 r_{\mathrm{c}}}{1 \mathrm{pc}}\right)^{0.38}<40 \sqrt{3} \frac{V_{0}^{2}}{s}$,

where $V_{0}=\sqrt{V^{2}+c_{\mathrm{s}}^{2}}$. Finally

$V_{0}>17 M_{10}^{0.138} \mathrm{~km} \mathrm{~s}^{-1}$.

Therefore for nearly any black hole velocity, interstellar medium turbulent motion cannot prevent realization of the spherical accretion regime ${ }^{1}$. Furthermore, density fluctuations cannot prevent it either. For $\frac{\Delta \rho}{\rho} \sim\left(\frac{r}{1 \mathrm{pc}}\right)^{\frac{11}{6}}$ (Armstrong et al. $1995)$, it may be easily shown that

$V_{0}>3.7 M_{10}^{0.39} \mathrm{~km} \mathrm{~s}^{-1}$.

It is clear that even in cold clouds, density fluctuations cannot lead to the disc accretion regime.

\subsection{Radial structure of the flow}

The general solution of hydrodynamical problem of accretion onto a non-moving gravitating center derived by Bondi (1952) determines radial profiles of various accretion flow parameters as a function of the distance to the black hole, accretion rate and gas adiabatic index. It is important for us that for $\gamma<5 / 3$ (that is always true for interstellar gas) there is a "sonic point" in the flow, passing which the gas motion becomes supersonic, and the gas velocity near the gravitating center has an asymptotic behaviour $v \propto r^{-1 / 2}$ (and so $\rho \propto r^{-3 / 2}$ for density). In an approximate description, we may extrapolate such a behaviour to the capture radius scale and use it for the whole flow.

In the black hole tail, where the matter flow stops, the thermal, gravitational and magnetic energy densities become nearly equal (since in the collisionless case, the matter is stopped due to the magnetic pressure and plasma oscillations, Illarionov \& Sunyaev 1975). This equipartition of energies (at least magnetic and gravitational ones) is preserved in the following infall (so-called Shvartsman equipartition theorem, Shvartsman 1971), so we may assume that (Bisnovatyi-Kogan \& Ruzmaikin 1974)

$\frac{B^{2}}{8 \pi}=\frac{1}{2} \rho v^{2}=\alpha^{2} \frac{G M \rho}{r}$,

where $\alpha^{2} \approx 1 / 3$ (which corresponds to equal amounts of gravitational energy transition into kinetic, magnetic and gravitational ones). Therefore, for the parameters of the accretion flow we have

$v=\alpha c \sqrt{\frac{r_{\mathrm{g}}}{r}}=\alpha c R^{-1 / 2}$,

\footnotetext{
${ }^{1}$ As already noticed, an obvious exception is the case of cold clouds where sonic speed is low and at $v<16-17 \mathrm{~km} \mathrm{~s}^{-1}$ a disk may form. We are planning to discuss this case in a separate paper.
}

$$
\begin{aligned}
& \rho=\frac{\dot{M}}{4 \pi r^{2} v}=\frac{m_{\mathrm{p}} \dot{m}}{2 \sigma_{\mathrm{T}} \alpha r_{\mathrm{g}}} R^{-3 / 2}, \\
& \frac{B^{2}}{8 \pi}=\frac{1}{2} \rho v^{2}=\frac{\alpha m_{\mathrm{p}} c^{2} \dot{m}}{4 \sigma_{\mathrm{T}} r_{\mathrm{g}}} R^{-5 / 2},
\end{aligned}
$$

where the dimensionless values for radius $R=r / r_{\mathrm{g}}$ and the accretion rate from Eq. (4) are used. Numerical values of these parameters are

$$
\begin{aligned}
& n=\frac{\rho}{m_{\mathrm{p}}}=4.33 \times 10^{12} \dot{m}_{-5} M_{10}^{-1} R^{-3 / 2} \mathrm{~cm}^{-3}, \\
& B=8 \times 10^{4} \dot{m}_{-5}^{1 / 2} M_{10}^{-1 / 2} R^{-5 / 4} \text { Gauss. }
\end{aligned}
$$

The magnetic field has a quasi-radial sectorial structure (the radial component grows much faster than the tangential one, which is proportional to the square root of distance; magnetic field lines are stretched).

The assumption introduced earlier on the equipartition of magnetic, kinetic and gravitational energy requires the existence of magnetic flux (and, therefore magnetic energy) dissipation mechanisms. It was first noted by Shvartsman (1971); their possible observational appearances were discussed by Illarionov \& Sunyaev (1975); a model-independent estimation of the energy dissipation rate was proposed by Bisnovatyi-Kogan \& Ruzmaikin (1974) and from a different point of view by Meszaros (1975); alternative approaches were discussed by Scharlemann (1983) and Kowalenko \& Melia (1999).

The dissipation rate of such a mechanism may be estimated as follows (Bisnovatyi-Kogan \& Ruzmaikin 1974). Scaling laws for the magnetic field in a given volume element for the frozen-in field and equipartition are correspondingly

$$
\begin{aligned}
& \left(\frac{\mathrm{d}}{\mathrm{d} t} \frac{B^{2}}{8 \pi}\right)_{\text {frozen-in }}=-4 \frac{v}{r} \frac{B^{2}}{8 \pi}, \\
& \left(\frac{\mathrm{d}}{\mathrm{d} t} \frac{B^{2}}{8 \pi}\right)_{\text {equipartition }}=-\frac{5}{2} \frac{v}{r} \frac{B^{2}}{8 \pi}, \\
& \frac{B^{2}}{8 \pi}=\frac{1}{2} \rho v^{2}=\alpha^{2} \frac{G M \rho}{r} .
\end{aligned}
$$

For the preservation of equipartition state, the power equal to the difference of these expressions must be dissipated in a volume element:

$\frac{\mathrm{d} E}{\mathrm{~d} V \mathrm{~d} t}=\frac{3}{2} \frac{v}{r} \frac{B^{2}}{8 \pi}$

or the same for a spherical shell

$\frac{\mathrm{d} E}{\mathrm{~d} R \mathrm{~d} t}=\frac{3 \alpha^{2}}{4} \frac{\dot{M} c^{2}}{R^{2}}$

which, by integrating over the whole volume, gives

$\frac{\mathrm{d} E}{\mathrm{~d} t}=\frac{3}{4} \alpha^{2} \dot{M} c^{2}=\frac{1}{4} \dot{M} c^{2}$,

which means that in the case of equipartition for the spherical flow described earlier, as much as $25 \%$ of infalling matter rest energy is released through this dissipation mechanism only. This leads to an additional super-adiabatic heating of the gas (Bisnovatyi-Kogan \& Ruzmaikin 1974; Meszaros 1975; Ipser \& Price 1977), and unavoidably changes the temperature radial profile and the luminosity of the accretion flow. 


\subsection{Flaring dissipation of the magnetic field and electron acceleration in the current sheets}

It is clear from simple physical reasons that dissipation of the magnetic field means that it is no longer frozen-in, i.e. the mean conductivity becomes much lower and relative motion of the magnetic field and plasma appears (the mean diffusion time becomes comparable to the free-fall one), currents begin to flow and heat the gas. These processes may take place either continuously in turbulent accretion flow as an Ohmic dissipation of the magnetic field (Bisnovatyi-Kogan \& Ruzmaikin 1974; Bisnovatyi-Kogan \& Lovelace 1997) or in compact enough separate regions. In the latter case, the dissipation process has a highly discrete nature (this possibility was noted by BisnovatyiKogan \& Lovelace 2000) of single events of magnetic field line reconnections.

In the present work we consider the latter case. It seems to be more realistic due to the fact that Alfven velocity which determines the speed of energy exchange between such a reconnection region and the surrounding plasma is nearly equal to the free-fall velocity at which a high magnetic field gradient forms. This is analogous to the case of continuous energy supply to the magnetic field inhomogeneities in the solar corona and its flaring (discrete) dissipation and conversion to acceleration of particles and anomalous turbulent heating. This is the case of formation of turbulent current sheets, which leads to fast magnetic field line reconnection, avalanche-like growth of energy release (the flare itself) and threshold switch-off of energy dissipation processes (Sweet 1969; Petchek 1964; Parker 1979; Spitzer 1954; Syrovatskii 1981). Multi-frequency observations of solar flares support this mechanism. On the other hand, the similarity of statistical properties of flaring activity of the Sun (Lu \& Hamilton 1991; Lu et al. 1993) and UV Cet stars (Gershberg 1989), X-ray binaries (Kawaguchi \& Mineshige 1999) gamma-ray bursts and active galactic nuclei argues in favor of the universality of such processes. So, we may assume that the main mechanism providing magnetic energy dissipation in the accretion flow is a reconnection of the magnetic field lines in the current sheets (regions of high magnetic field gradient).

Energy dissipation in the current sheet itself may be considered as a simple Joule heating $Q=j E=j^{2} / \sigma$, where $j=(c / 4 \pi) \cdot \operatorname{rot} \boldsymbol{B} \propto \frac{\Delta B}{a}-$ current density, $a-$ current sheet thickness, $\sigma$ - some effective conductivity. When current reaches some threshold value, $j_{\mathrm{c}}=e n_{\mathrm{c}} c_{\mathrm{i}}$, where $n_{\mathrm{c}}$ - electron number density, $c_{\mathrm{i}}$ - ion sound speed, it generates strong ionacoustic turbulence that lowers the conductivity by $9-10$ orders of magnitude (electrons begin to collide with plasmons and heat them). The analysis of particle acceleration in such a current sheet (which may be considered as a superposition of direct electric field acceleration and diffusion - elastic scattering on plasmons) was performed in Pustilnik $(1978,1997)$. In the framework of this model, the accelerated particle energy distribution (electrons mostly) naturally appears to be a power-law up to energies large enough (Pustilnik 1978) and has a shape

$f_{0}(\gamma)=\frac{1}{\Gamma}\left(\frac{\Gamma}{\gamma}\right)^{3} \mathrm{e}^{-\frac{\Gamma}{\gamma}}$ where $\Gamma$ corresponds to the mean energy of a particle in a beam.

We estimate the maximal energy a electron reaches by acceleration in such current sheet. By using expressions for gas density and magnetic field strength (13), (14) for low accretion rates $\left(\dot{m}<10^{-5}\right)$, we have for the event horizon $n \leq$ $4 \times 10^{12} \mathrm{~cm}^{-3}$ and $B_{\mathrm{g}} \leq 1.7 \times 10^{5}$ Gs. Near $r_{\mathrm{g}}$ velocities of electrons and plasmons are nearly equal to the speed of light, and so, the maximum current density is $j_{\max }=e n c$, and the maximum effective field is $E_{\max }=\frac{e n c}{\sigma^{*}}$, where $\sigma^{*}$ is anomalous resistivity; for our case $\sigma^{*} \sim \frac{10^{2}}{4 \pi} \omega_{\mathrm{oe}}$, where $\omega_{\mathrm{oe}}-$ Lengmure frequency $\left(\omega_{\mathrm{oe}}=5.65 \times 10^{4} \cdot n^{1 / 2}\right)$. Therefore, the maximum gamma-factor is

$\gamma_{\max } \sim \frac{e E_{\max } r_{\mathrm{g}}}{m_{\mathrm{e}} c^{2}} \sim \frac{e^{2} n r_{\mathrm{g}}}{\sigma^{*} m_{\mathrm{e}} c} \sim \frac{e^{2} r_{\mathrm{g}} n^{1 / 2}}{4.5 \times 10^{5} m_{\mathrm{e}} c} \sim 10^{5}$.

It is very difficult to estimate the fraction $\xi$ of the dissipated energy carried away by the accelerated particles - electrons during the acceleration process generate ion-acoustic and Lengmure plasma oscillations. Also, topology changes in reconnections lead to global matter motions. A study of such processes in the solar flares shows that particles (electrons mostly) carry away from $10 \%$ to $50 \%$ of energy stored in the magnetic field inhomogeneities (Hudson \& Ryan 1995). We assume $\xi=0.1$ as a reasonable lower limit for further estimations. The $(1-\xi)$ fraction of the dissipated energy goes into the surrounding plasma heating. In the collisionless case this is due to generation of MHD turbulence in the current sheet. Also, as the speed of plasmons is nearly equal to the Alfven speed, which in turn is equal to the free-fall velocity for the equipartition case, the heating is nearly uniform through the whole accretion flow. It is also difficult to determine the motion of the accelerated particle beam. Of course, electrons are moving along the magnetic field lines, tracing its topology. Its motion is local due to the relative smallness of Larmor radius in comparison to the characteristic scale of the accretion flow, Schwarzschild radius $r_{\mathrm{g}}$

$\left(\frac{r_{\mathrm{L}}}{r_{\mathrm{g}}}\right)^{2}=\frac{\gamma^{2}}{\alpha \dot{m}} \frac{m_{\mathrm{e}}}{m_{\mathrm{p}}} \sqrt{\frac{2 \sigma_{\mathrm{T}}}{3 \pi r_{\mathrm{g}}^{2}}} \ll 1$.

For a typical single stellar-mass black hole accretion rates, electron-electron and electron-ion energy exchange is highly ineffective (this question has been discussed in detail in the framework of advective disk models, see Mahadevan \& Quataert 1997 and Bisnovatyi-Kogan \& Lovelace 2001), so we may neglect collisional energy losses for the accelerated particles.

Furthermore, in the case of a Maxwellian distribution of the background plasma electrons and moderate mean gammafactors of the accelerated nonthermal electrons $\left(10-10^{2}\right.$ of the mean thermal one), the total electron distribution $f(\gamma)$ always (for energies above thermal peak) satisfies the inequality $\frac{\mathrm{d} f(\gamma)}{\mathrm{d} \gamma}<0$, and so is stable to the generation of plasmons with wave vectors parallel to the motion direction (Kaplan \& Tsytovich 1973). For the same reason, all generated instabilities vanish rapidly and the beam is stabilized. However, the induced generation of plasmons with non-collinear wave vectors is possible; this leads to the dissipation of the beam with 


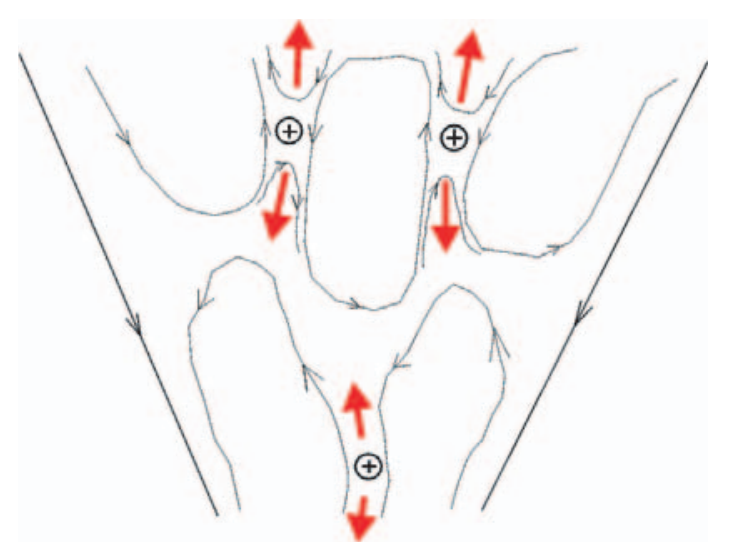

Fig. 2. A schematic picture of the accretion flow structure. Reconnection regions and ejected plasma flows are shown. The direction of the particles acceleration (current flow) is perpendicular to the viewing plane.

a characteristic time of $t_{\mathrm{dis}} \sim 10^{2} \frac{n\langle\gamma\rangle}{n_{*} \omega_{\mathrm{oc}}}$, where $n_{*}$ is the electron density of the beam (Kaplan \& Tsytovich 1973). It is easily seen that $\frac{t_{\text {dis }}}{t_{\mathrm{ff}}} \sim r^{-3 / 4}$, so, the beam born near $r_{\mathrm{g}}$ has more chance of surviving. Also it is seen that for $\frac{n}{n_{*}}>10^{2}$ the beam has not enough time to dissipate in the free-fall time scale, so, we may neglect this scattering and thermalization of the beam and consider it as a system of non-interacting electrons in the external magnetic field.

The magnetic field dissipation in the current sheet is accompanied by significant dynamical effects; the reconnected magnetic field lines are ejected through the current sheet ends carrying away the gas that lowers the current sheet density (see Fig. 2).

The results of the solar corona and chromosphere observations (Dere 1996; Innes et al. 1997) show that the reconnection speed is often significantly less than the Alfven one $\left(\approx 0.1 V_{\mathrm{A}}\right)$. In such a case the density and temperature fluctuations may not have enough time to vanish, and this may lead to additional variability of the accretion flow.

The magnetic energy dissipation mechanism proposed here is not a unique one. However, it is in a good agreement with the observations of the Sun (Pustilnik 1997). Also, it provides an opportunity for interpretation of the universal energetic spectrum of flares on various objects - from active galactic nuclei to UV-Cet stars in the framework of a single mechanism.

To complete the picture, we briefly review other models. Fast reconnections leading to particle acceleration were considered by Lazarian \& Vishniac $(1999,2000)$, the role of various instabilities in creation of anomalous resistivity and particles acceleration has been considered by Birk et al. (1999), and processes of particle acceleration in various astrophysical objects by Bisnovatyi-Kogan \& Lovelace (1997, 2000, 2001).

The dissipated magnetic energy is converted mostly to acceleration of electrons. In the framework of our paper this means that for analysis of emission processes we may take into account the electron component only.

\section{Derivation of electron distribution}

\subsection{Note on adiabatic heating}

At the low accretion rates considered, the gas is fully collisionless, and so the particle energy distribution is determined by superposition of three different factors - adiabatic heating, synchrotron cooling and nonthermal heating due to particle acceleration in the current sheets. The former dominates at large distances, and so its precise treatment is very important.

At the level of single particle motion, adiabatic heating of collisionless magnetized gas is due to the conservation of the adiabatic invariant (Landau \& Lifshitz 1971)

$I=\frac{3 c p_{\mathrm{t}}^{2}}{2 e B}$

of the charged particle in the magnetic field evolving slowly on a timescale of a single Larmor revolution. This basically means the conservation of the phase volume element per particle $p_{\mathrm{t}}^{3}$. $V=$ const. Here we neglect the motion parallel to the magnetic field line - only the perpendicular momentum $p_{\text {t }}$ grows due to compression, while the parallel one $p_{\|}$is determined mostly by the initial conditions.

Note that classical relation between the spatial part of the phase volume and the gas density $V \rho=$ const., that is true for normal "gas in a box" of classical thermodynamics, is generally wrong for magnetized plasma. An exception is the case of isotropic gas compression with $B \propto l^{-2} \propto \rho^{2 / 3}$ - in this case the magnetic field acts as the walls of a box, and this leads to the usual equations of state $\epsilon \propto \rho^{5 / 3}$ for the non-relativistic case or $\epsilon \propto \rho^{4 / 3}$ for relativistic one. Here we use the proportionality of the particle energy to its perpendicular momentum, that is true for two opposite cases - either for $p_{\|} \propto p_{\mathrm{t}}$ (effective isotropization) or for $p_{\|} \ll p_{\mathrm{t}}$ (collisionless case, only perpendicular momentum increases).

In the case of the accretion flow, however, the gas compression is significantly anisotropic (the matter element is even stretched in the radial direction proportional to $r^{-1 / 2}$ ), and the magnetic field itself is not perfectly frozen-in either. So, the relation of the phase volume element spatial part to the density becomes

$\frac{\mathrm{d} V}{V}=-3 \frac{\mathrm{d} p_{\mathrm{t}}}{p_{\mathrm{t}}}=-\frac{3}{2} \frac{\mathrm{d} B}{B}=-\frac{15}{8} \frac{\mathrm{d} r}{r}=-\frac{5}{4} \frac{\mathrm{d} \rho}{\rho}=-\frac{5}{4} \frac{\mathrm{d} n}{n}$,

where the radial dependencies (13), (14) of the accretion flow parameters are used.

Note that in contrary to assumptions made in most articles on this subject (Bisnovatyi-Kogan \& Ruzmaikin 1974; Shapiro 1973b; Meszaros 1975; Ipser \& Price 1977, 1982; Mahadevan \& Quataert 1997) we cannot use the first law of thermodynamics in the form of "energy per particle"

$\mathrm{d}\left(\frac{\epsilon}{n}\right)=-p \mathrm{~d}\left(\frac{1}{n}\right)+\ldots$

(the dots here represent the contribution of non-adiabatic processes) to describe the relation of the particle mean energy and the gas density as the density changes don't reflect the behaviour of the particle "walls". A correct form of this equation must take into account the fact that the number of particles $N$ 
inside the spatial part of each particle conserving phase volume element is no longer constant

$\mathrm{d}(\epsilon V)=-p \mathrm{~d} V+\frac{\epsilon}{n} \mathrm{~d} N+\ldots$

Using (27), the change of the number of particles may be expressed as

$\mathrm{d} N=n V\left(\frac{\mathrm{d} V}{V}-\frac{\mathrm{d}(1 / n)}{(1 / n)}\right)=-\frac{1}{4} V \mathrm{~d} n$.

For relativistic gas the equation of state has the form $p=\epsilon / 3$, and so the single particle energy evolution is described by

$\mathrm{d}\left(\frac{\epsilon}{n}\right)=-\frac{5}{12} \epsilon \mathrm{d}\left(\frac{1}{n}\right)+\ldots$,

where dots represent the possible contribution of non-adiabatic processes per particle (thus, such particles behave like a gas with specific heat ratio $11 / 6$ ).

For non-relativistic gas in a similar manner $\left(p=\frac{2}{3} \epsilon\right)$

$\mathrm{d}\left(\frac{\epsilon}{n}\right)=-\frac{5}{6} \epsilon \mathrm{d}\left(\frac{1}{n}\right)+\ldots$

like the gas with a specific heat ratio of $17 / 12$.

Equations (32) and (31) may be rewritten in a form useful for comparison with the incorrect expression (28):

$\mathrm{d}\left(\frac{\epsilon}{n}\right)=-\frac{5}{4} p \mathrm{~d}\left(\frac{1}{n}\right)+\ldots$

It is easily seen that correct consideration of adiabatic heating makes it $25 \%$ more effective than in the case of ideal nonmagnetized gas accretion of Bondi (1952), that has a large influence on luminosity and spectral shape of the accretion flow emission.

\subsection{Radial temperature distribution}

Note that adiabatic heating alone does not change the shape of the particle momentum distribution, so initially thermal distribution always stays thermal.

Current sheet spatial scales are usually much smaller than the whole accretion flow one and, therefore, the fraction of the accelerated particles is small, and so the total (significantly nonthermal) electron distribution may be considered as a superposition of the purely thermal one for the background flow particles and purely nonthermal for the ones accelerated in the current sheets (this is the approach known as "hybrid plasma" of Coppi 1999). Also we assume that for low accretion rates the non-adiabatic heating and radiative energy losses do not change the shape of the thermal component distribution (while changing its mean energy)

$f(R, \gamma)=f_{\mathrm{t}}(R, \gamma)+\zeta f_{\mathrm{nt}}(R, \gamma)$.

Note that this distribution is not normalized, and only its shape has physical meaning. So, for example, the ratio of nonthermal to thermal electron densities at some radius $R$ may be expressed as

$\frac{n_{\mathrm{nt}}(R)}{n_{\mathrm{t}}(R)}=\frac{\zeta f_{\mathrm{nt}}(R)}{f_{\mathrm{t}}(R)}$ where $f_{\mathrm{nt}}(R)$ and $f_{\mathrm{t}}(R)$ are integrals of the corresponding distribution functions over the range of $\gamma$.

Thermal particle distribution function may be written as

$f_{\mathrm{t}}(R, \gamma)=\frac{\sqrt{R}}{2 \tau}\left(\frac{\gamma}{\tau}\right)^{2} \exp \left(-\frac{\gamma}{\tau}\right)$

where the usual dimensionless expression for temperature $\tau=$ $k T / m_{\mathrm{e}} c^{2}$ is used. This gives a Maxwellian local energy distribution and radial density slope $\rho \propto R^{-3 / 2}$.

The temperature distribution $\tau(R)$ may be determined by solving the energy balance equation taking into account heating due to adiabatic compression and magnetic field dissipation and radiative losses. Note that electrons become relativistic at some large radius $R_{\text {rel }}$, while protons remain non-relativistic until the event horizon (Bisnovatyi-Kogan \& Ruzmaikin 1974), and so their heating rates differ by a factor of $2(31,32)$. In the case of ineffective energy exchange (at low accretion rates collisions are very rare; other mechanisms noted in Mahadevan \& Quataert 1997 are also ineffective); this could have led to electrons much colder than protons, but due to preferred heating of electrons by non-adiabatic processes (Bisnovatyi-Kogan \& Lovelace 2000,2001 ) its energies always remain roughly of the same order of magnitude. Of course their gamma-factors will differ by about 40 times, and so the main contribution to the accretion flow radiation is due to electrons. Therefore, only electron temperature is interesting for us.

As it has been noticed before (20), the non-adiabatic heating rate may be expressed as

$\Phi=(1-\xi) \frac{\mathrm{d} E}{\mathrm{~d} V \mathrm{~d} t}=(1-\xi) \frac{3}{2} \frac{v}{r} \frac{B^{2}}{8 \pi}$.

The main mechanism of radiative losses at low accretion rates is synchrotron radiation. Its rate may be written as (Lightman \& Rybicki 1979)

$\Lambda_{\text {sync }}=\frac{4}{3} \sigma_{\mathrm{T}} c \gamma^{2} \frac{B^{2}}{8 \pi} n$.

For a Maxwellian distribution

$\overline{\gamma^{2}}=12\left(\frac{k T}{m_{\mathrm{e}} c^{2}}\right)^{2}=12 \tau^{2}$.

Taking into account non-adiabatic terms, the energy balance equation per particle (32) may be written for a non-relativistic region of the flow $\left(R>R_{\text {rel }}\right)$ as

$\frac{\mathrm{d}}{\mathrm{d} r} \frac{\epsilon}{n}=-\frac{5}{4} \frac{\epsilon}{n r}+\frac{1}{v} \frac{\Lambda-\Phi}{n}$.

For a non-relativistic electron gas $\epsilon=\frac{3}{2} n k T$; also we may neglect energy losses and rewrite it in variables $R$ and $\tau$ as

$\frac{\mathrm{d} \tau}{\mathrm{d} R}=-\frac{5}{4} \frac{\tau}{R}-(1-\xi) \frac{\alpha^{2}}{2} \frac{m_{\mathrm{p}}}{m_{\mathrm{e}}} R^{-2}$.

This gives the value of the radius for $\tau=1, R_{\text {rel }} \approx 6400$ for $\xi=0.1$, that significantly exceeds $R_{\text {rel }} \approx 1300$ in the ideal gas approximation used by Bisnovatyi-Kogan \& Ruzmaikin (1974). 
In deeper regions, electrons become relativistic, their energy density is $\epsilon=3 p=3 n k T$, and so for $R<R_{\text {rel }}$ the energy balance Eq. (31) combined with (13), (14) and (4) gives

$\frac{\mathrm{d} \tau}{\mathrm{d} R}=-\frac{5}{8} \frac{\tau}{R}-(1-\xi) \frac{\alpha^{2}}{4} \frac{m_{\mathrm{p}}}{m_{\mathrm{e}}} R^{-2}+\frac{4}{3} \frac{m_{\mathrm{p}}}{m_{\mathrm{e}}} \frac{\dot{m} \tau^{2}}{R^{2}}$.

The boundary condition is $\tau\left(R_{\text {rel }}\right)=1$.

An analytical solution of this equation in general is very difficult, but for low accretion rates we may neglect the influence of radiative losses and get a solution in the form

$\tau(R)=(1-\xi) \frac{2 \alpha^{2}}{3} \frac{m_{\mathrm{p}}}{m_{\mathrm{e}}} R^{-1}+\left(1-(1-\xi) \frac{2 \alpha^{2}}{3 R_{\mathrm{rel}}} \frac{m_{\mathrm{p}}}{m_{\mathrm{e}}}\right)\left(\frac{R_{\mathrm{rel}}}{R}\right)^{5 / 8}$.

This expression may be substituted into (36) to get the final expression for the thermal electron distribution.

The applicability of this approximation may be estimated by comparing the timescales of the electron radiative energy losses and of the free-fall near the event horizon

$\frac{t_{\mathrm{ff}}}{t_{\mathrm{rad}}}=\frac{\alpha}{3} \frac{m_{\mathrm{p}}}{m_{\mathrm{e}}} \gamma \dot{m}<1 \quad$ for $\quad \dot{m}<10^{-5}$,

where $\gamma \approx 100$ is assumed. For higher accretion rates Eq. (42) may be easily solved numerically.

\subsection{Distribution function of the non-thermal component}

We can build an expression for nonthermal component distribution function. We may assume that all electrons are relativistic as only these have significant observational appearances, and electrons are relativistic in the most interesting regions near the horizon (well inside the relativization radius $R_{\text {rel }} \approx 6000$ ). So, the fraction of low-energy nonthermal particles is negligible, and nearly all electrons have a "lifetime" not less than the characteristic free-fall time scale (see Sect. 2.4).

So, we may neglect nonthermal particle interactions with electrons and plasmons of the background flow (see discussion in Sect. 2.4) and assume that they evolve due to adiabatic heating and synchrotron energy losses only. Note that this leads to the situation where thermal electron energy grows slightly faster than the nonthermal one due to additional heating by plasma oscillations ejected by current sheets.

The energy evolution of single nonthermal electron is described by

$\frac{\mathrm{d} \gamma}{\mathrm{d} R}=\frac{1}{3} \frac{m_{\mathrm{p}}}{m_{\mathrm{e}}} \dot{m} \frac{\gamma^{2}}{R^{2}}-\frac{5}{8} \frac{\gamma}{R}$,

where the first term corresponds to synchrotron losses and the second one to adiabatic heating.

For the initial energy $\gamma_{0}$ at $R_{0}$ it has the solution

$\gamma=\frac{\gamma_{0}}{C_{1}\left(R, R_{0}\right) \gamma_{0}+C_{2}\left(R, R_{0}\right)}$

where

$C_{1}\left(R, R_{0}\right)=\frac{A}{R}\left(1-\left[\frac{R}{R_{0}}\right]^{13 / 8}\right)$

$$
\begin{aligned}
& A=\frac{8}{39} \frac{m_{\mathrm{p}}}{m_{\mathrm{e}}} \dot{m} \\
& C_{2}\left(R, R_{0}\right)=\left(\frac{R}{R_{0}}\right)^{5 / 8} .
\end{aligned}
$$

The nonthermal component at some radius $R$ consists of noninteracting electron beams generated at all radii $R_{0}>R$. The evolution of the distribution function of each such beam (assuming that the ejection process is stationary and the initial beam distribution function is $\left.f_{\mathrm{b}}\left(R_{0}, \gamma_{0}\right)\right)$ is as follows:

$$
\begin{aligned}
f_{\mathrm{b}}(R, \gamma) & =f_{\mathrm{b}}\left(R_{0}, \gamma_{0}\right) \frac{\mathrm{d} \gamma_{0}}{\mathrm{~d} \gamma} \\
& =f\left(R_{0}, \gamma_{0}\right) \frac{\left(C_{1}\left(R, R_{0}\right) \gamma_{0}+C_{2}\left(R, R_{0}\right)\right)^{2}}{C_{2}\left(R, R_{0}\right)} .
\end{aligned}
$$

The initial beam distribution has a form (see Eq. (23))

$f_{\mathrm{b}}\left(R_{0}, \gamma_{0}\right)=\frac{f_{\mathrm{b}}\left(R_{0}\right)}{\Gamma\left(R_{0}\right)}\left(\frac{\Gamma\left(R_{0}\right)}{\gamma_{0}}\right)^{3} \exp \left(-\frac{\Gamma\left(R_{0}\right)}{\gamma_{0}}\right)$,

where the mean energy

$\overline{\gamma_{0}}=\Gamma=\Delta \tau$

is assumed to be by a fixed factor $\Delta$ greater than the local thermal component one and $f_{\mathrm{b}}(R)$ describes the radial distribution of the accelerated particle ejection rate $\mathrm{d} N / \mathrm{d} t$. The latter may be computed using Eq. (21) as

$\frac{\mathrm{d} N}{\mathrm{~d} R \mathrm{~d} t}=\frac{1}{m_{\mathrm{e}} c^{2} \Gamma(R)} \frac{\xi \mathrm{d} E}{\mathrm{~d} R \mathrm{~d} t}=\frac{6 \pi \alpha^{2}}{4} \frac{m_{\mathrm{p}}}{m_{\mathrm{e}}} \frac{r_{\mathrm{g}} c}{\sigma_{\mathrm{T}}} \frac{\xi \dot{m}}{R^{2} \Gamma(R)}$,

which gives for the total ejection rate

$\frac{\mathrm{d} N}{\mathrm{~d} t}=\frac{6 \pi \alpha^{2}}{4} \frac{m_{\mathrm{p}}}{m_{\mathrm{e}}} \frac{r_{\mathrm{g}} c}{\sigma_{\mathrm{T}}} \xi \dot{m} \int_{1}^{\infty} \frac{\mathrm{d} R}{R^{2} \Gamma(R)}$,

and the radial distribution of particle ejection

$f_{\mathrm{b}}\left(R_{0}\right)=\frac{a_{0}}{\Gamma\left(R_{0}\right) R_{0}^{2}}$, where $a_{0}=\left(\int_{1}^{\infty} \frac{\mathrm{d} R_{0}}{\Gamma\left(R_{0}\right) R_{0}^{2}}\right)^{-1}$.

By integrating Eq. (47) over all $R_{0}>R$ we may get final expression for nonthermal electron distribution which consists of all beam electrons ejected at greater distances

$$
\begin{aligned}
f_{\mathrm{nt}}(R, \gamma)= & \int_{R}^{\infty} \frac{a_{0} \mathrm{~d} R_{0}}{R_{0}^{2}} \frac{\Gamma\left(R_{0}\right)}{\gamma_{0}^{3}} \frac{\left[C_{1}\left(R, R_{0}\right) \gamma_{0}+C_{2}\left(R, R_{0}\right)\right]^{2}}{C_{2}\left(R, R_{0}\right)} \\
& \times \exp \left(-\frac{\Gamma\left(R_{0}\right)}{\gamma_{0}}\right) .
\end{aligned}
$$

Now we determine the $\zeta$ coefficient of the total electron distribution (34). By combining (35) with trivial expressions for the energy density ratio

$\frac{\epsilon_{\mathrm{nt}}(R)}{\epsilon_{\mathrm{t}}(R)}=\frac{n_{\mathrm{nt}}(R)}{n_{\mathrm{t}}(R)} \frac{\bar{\gamma}_{\mathrm{t}}(R)}{\bar{\gamma}_{\mathrm{nt}}(R)}$

and for the energy density of thermal electrons with a Maxwellian value $\bar{\gamma}_{\mathrm{t}}(R)=3 \tau(R)$

$\epsilon_{\mathrm{t}}=3 m_{\mathrm{e}} c^{2} \tau(R) n_{\mathrm{t}}$ 


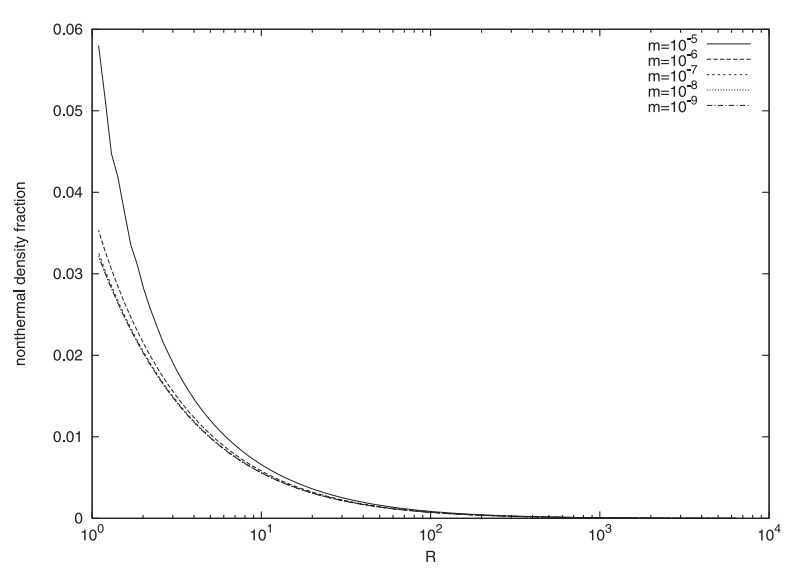

Fig. 3. Nonthermal electron density fraction $n_{\mathrm{nt}}(R) / n(R)$ for an $M=$ $10 M_{\odot}$ black hole, $\xi=0.1$ and the number of accretion rates.

we may get

$\zeta=\frac{\epsilon_{\mathrm{nt}}(R) \sqrt{R}}{m_{\mathrm{e}} c^{2} n_{\mathrm{t}}(R)}\left(\int_{1}^{\infty} \gamma f_{\mathrm{nt}}(R, \gamma) \mathrm{d} \gamma\right)^{-1}$.

The fraction of nonthermal particles is always small, so we may replace here the thermal particle number density $n_{\mathrm{t}}(r)$ with the total one $n(R)$.

The nonthermal electron energy density at $R$ may be computed by considering an elementary spherical shell $R \div R+\delta R$ and integrating the nonthermal energy release in it during its whole backtrace free-fall history

$\epsilon_{\mathrm{nt}}(R)=\frac{1}{4 \pi r_{\mathrm{g}}^{3} R^{2} \delta R} \int_{R}^{\infty} \frac{\delta R_{0} \mathrm{~d} R_{0}}{-\frac{\mathrm{d} R_{0}}{\mathrm{~d} t}}\left(\frac{\xi \mathrm{d} E}{\mathrm{~d} R_{0} \mathrm{~d} t}\right) \frac{\bar{\gamma}_{\mathrm{b}}(R)}{\Gamma\left(R_{0}\right)}$,

where the latter multiplicative term corresponds to amplification of the mean gamma-factor of each accelerated electron beam ejected at $R_{0}$ in free-fall until $R$. This quantity may be written using the results of Appendix A as

$\frac{\bar{\gamma}_{\mathrm{b}}(R)}{\Gamma\left(R_{0}\right)}=\mathfrak{F}_{1}\left(\left(\frac{R}{R_{0}}\right)^{5 / 8}, \frac{A \Gamma\left(R_{0}\right)}{R}\left(1-\left[\frac{R}{R_{0}}\right]^{13 / 8}\right)\right)$.

This combined with the expression for the local nonthermal energy dissipation rate (21) and with the scaling for the given spherical shell thickness at free-fall $\delta R_{0} \propto R_{0}^{-1 / 2}$ leads to the final expression for the $\zeta$ coefficient

$$
\begin{aligned}
\zeta= & \frac{3}{4} \frac{m_{\mathrm{p}}}{m_{\mathrm{e}}} \xi \alpha^{2} \sqrt{R}\left(\int_{1}^{\infty} \gamma f_{\mathrm{nt}}(R, \gamma) \mathrm{d} \gamma\right)^{-1} \\
& \times \int_{R}^{\infty} \frac{\mathrm{d} R_{0}}{R_{0}^{2}} \Im_{1}\left(\left(\frac{R}{R_{0}}\right)^{5 / 8}, \frac{A \Gamma\left(R_{0}\right)}{R}\left(1-\left[\frac{R}{R_{0}}\right]^{13 / 8}\right)\right) .
\end{aligned}
$$

A sample fraction of the nonthermal to total densities for different accretion rates is shown in Fig. 3 as a function of distance from the black hole. This fraction is small. Figure 4 shows the shapes of thermal and non-thermal distributions for two different distances at low accretion rates.
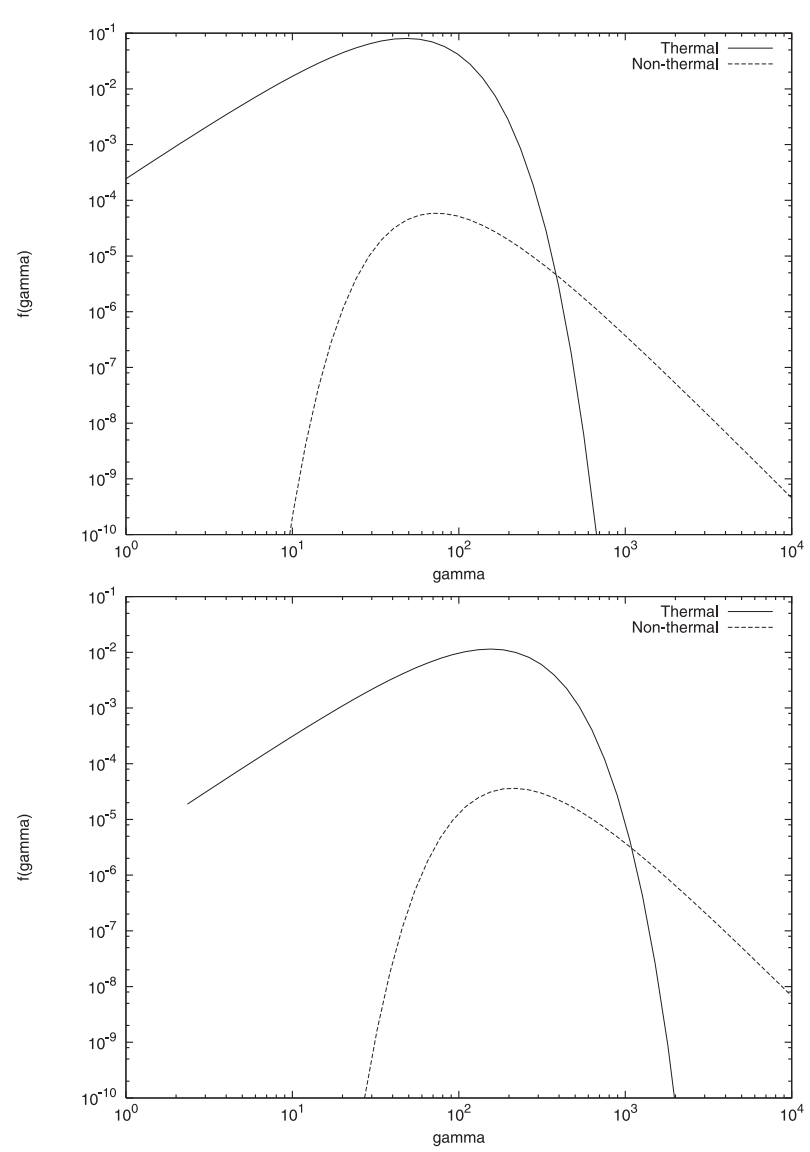

Fig. 4. Energetic distributions of thermal $f_{\mathrm{t}}(R, \gamma)$ and nonthermal $f_{\mathrm{nt}}(R, \gamma)$ electron components for distances of $R=50 r_{\mathrm{g}}$ (first panel) and $R=10 r_{\mathrm{g}}$ (second panel), respectively, for $\Delta=10$.

\section{Emission spectrum}

The emission spectrum of a single electron for an observer at infinity has the shape (Lightman \& Rybicki 1979; Shapiro 1973a)

$L_{v}=2 \pi \int_{-1}^{\cos \theta^{*}} j_{v^{\prime}} \frac{1-\beta^{2}}{(1-\beta \cos \theta)^{2}} \mathrm{~d} \cos \theta$,

where relativistic effects of time contraction, gravitational redshift, Doppler effect and the capture of some emission fraction by the event horizon are taken into account. The event horizon angular size for a free-falling emitter is

$\left|\cos \theta^{*}\right|=\sqrt{1-\frac{27}{4 R^{2}}\left(1-\frac{1}{R}\right)}$,

where $\cos \theta^{*}<0$ for $R<1.5$. The quantity

$\beta=\frac{\mathrm{d} r}{\mathrm{~d} t} \frac{1}{1-r_{\mathrm{g}} / r}=\frac{v / c}{\left(v^{2} / c^{2}+1-r_{\mathrm{g}} / r\right)^{1 / 2}}$

represents the falling velocity of matter in the distant observer frame, and the frequency shift is given by

$v^{\prime}=v \frac{1-(v / c) \cos \theta}{\sqrt{\left(1-v^{2} / c^{2}\right)(1-1 / R)}}$. 


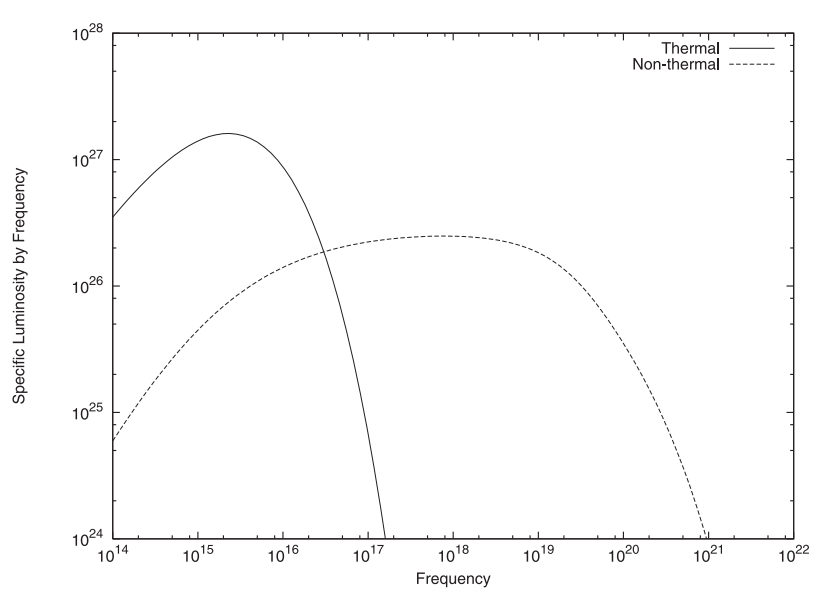

Fig. 5. Decomposition of a single black hole (with the mass $10 M_{\odot}$ ) emission spectrum into thermal and nonthermal parts. The accretion rate is $1.4 \times 10^{10} \mathrm{~g} / \mathrm{s}$, which corresponds to $\dot{m}=10^{-8}$, and $\xi=0.1$.

By substituting synchrotron emissivity from Lightman \& Rybicki (1979) we get

$j_{v}=\frac{\sqrt{3} e^{3} B \sin \psi}{4 \pi m_{\mathrm{e}} c^{2}} F\left(\frac{\nu^{\prime}}{\nu_{\mathrm{c}}}\right)$,

$F(x)=x \int_{x}^{\infty} K_{5 / 3}(\xi) \mathrm{d} \xi$

$v_{\mathrm{c}}=\frac{3 \gamma^{2} e B \sin \psi}{4 \pi m_{\mathrm{e}} c}$

Taking into account the fact that adiabatic compression increases the perpendicular electron momentum only (and so we may take $\sin \psi \approx 1$ for the pitch-angle) and convolving with the electron distribution (34), the final expression for the accretion flow emission spectrum is

$L_{v} \propto \int_{1}^{\infty} R^{-5 / 4} \mathrm{~d} R \int_{1}^{\infty} f(R, \gamma) \int_{-1}^{\cos \theta^{*}} \frac{1-\beta^{2}}{1-\beta \cos \theta} F\left(\frac{v^{\prime}}{\nu_{\mathrm{c}}}\right) \mathrm{d} \cos \theta \mathrm{d} \gamma$

Figure 5 shows a decomposition of the accretion flow spectrum into thermal and nonthermal parts. The fraction of the nonthermal emission is small in the optical range, but dominates in harder spectral bands. The spectrum becomes flatter with the accretion rate decrease due to the increase of the electron radiative loss timescale. The high-energy spectral cut-off is determined by radiative energy losses and by the upper limits of the accelerated electron gamma-factor (see Fig. 6).

\section{Luminosity}

The luminosity of the accretion flow may be computed by integrating expression (63) (Shapiro 1973a; Ipser \& Price 1977)

$$
\begin{aligned}
L= & 8 \pi^{2} \int_{1}^{\infty} R^{2} \mathrm{~d} R \int_{-1}^{\cos \theta^{*}} \int_{0}^{\infty} j_{v^{\prime}} \mathrm{d} v^{\prime} \\
& \times\left(1-\frac{r_{\mathrm{g}}}{r}\right)^{1 / 2}\left(1-\beta^{2}\right)^{3 / 2}(1-\beta \cos \theta)^{-3} \mathrm{~d} \cos \theta .
\end{aligned}
$$

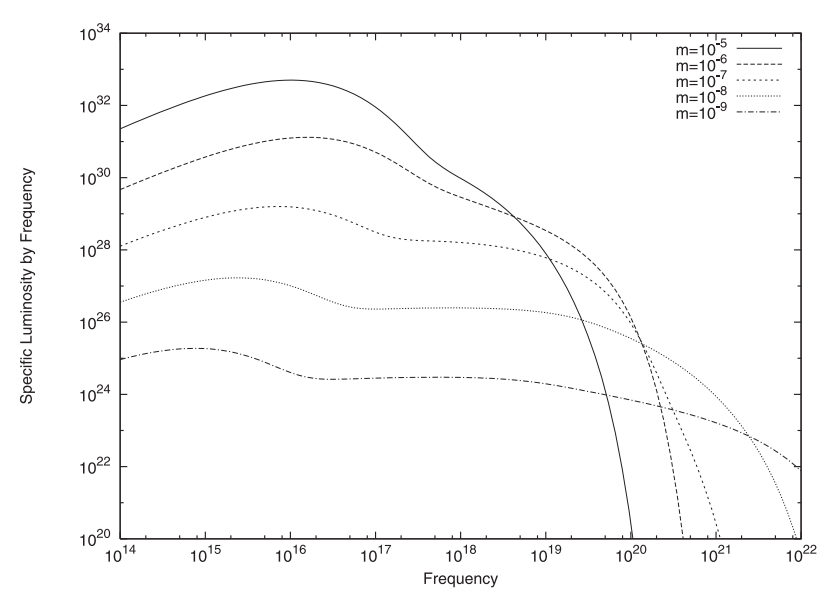

Fig. 6. Accreting $10 M_{\odot}$ black hole spectra for accretion rates from $\dot{m}=10^{-9}$ till $\dot{m}=10^{-5}\left(\dot{M}=1.4 \times 10^{9} \mathrm{~g} / \mathrm{s}\right.$ and $\dot{M}=1.4 \times 10^{13} \mathrm{~g} / \mathrm{s}$, correspondingly), for $\xi=0.1$.

Dividing by $\dot{M} c^{2}$ and taking into account the expression for synchrotron luminosity of a single electron (38), we get the expression for the efficiency of the thermal component emission

$\eta_{t}=4 \dot{m} \int_{1}^{\infty} \frac{\tau^{2}}{R^{2}} K(R) \mathrm{d} R$

where

$K(R)=\frac{1}{2} \int_{-1}^{\mu^{*}} \frac{(1-1 / R)^{1 / 2}\left(1-\beta^{2}\right)^{3 / 2}}{(1-\beta x)^{3}} \mathrm{~d} x$.

At low accretion rates $\left(\dot{m} \ll 10^{-5}\right)$ a reasonably good approximation for this quantity is

$\eta_{\mathrm{t}}=4.5 \times 10^{4} \dot{m}$

which corresponds to the thermal luminosity of

$L_{\mathrm{t}}=9.6 \times 10^{33} M_{10}^{3} n_{1}^{2}\left(V^{2}+c_{\mathrm{s}}^{2}\right)_{16}^{-3} \mathrm{erg} / \mathrm{s}$.

The efficiency of the nonthermal component emission may be easily estimated from the radial distribution of the dissipated magnetic energy $\mathrm{d} E / \mathrm{d} R \mathrm{~d} t$, its fraction $\xi$ carried out by accelerated particles and the evolution of its mean square of the gamma-factor during the fall towards the horizon $\bar{\gamma}_{\mathrm{b}}^{2}(R)$ as

$\eta_{\mathrm{nt}}=\frac{\xi}{4} \int_{1}^{\infty} \frac{a_{0} \mathrm{~d} R_{0}}{\Gamma^{2}\left(R_{0}\right) R_{0}^{2}} \int_{1}^{R_{0}}\left(\frac{\mathrm{d} \bar{\gamma}_{\mathrm{b}}}{\mathrm{d} R}\right)_{\mathrm{emis}} K(R) \mathrm{d} R$,

where the mean radiative energy losses of a single electron are

$\left(\frac{\mathrm{d} \bar{\gamma}_{\mathrm{b}}}{\mathrm{d} R}\right)_{\mathrm{emis}}=\frac{1}{3} \frac{m_{\mathrm{p}}}{m_{\mathrm{e}}} \dot{m} \frac{\overline{\gamma_{\mathrm{b}}^{2}}}{R^{2}}$

The evolution of the mean square of gamma-factor $\bar{\gamma}_{\mathrm{b}}^{2}(R)$ of the beam is given by expression (A.4) derived in Appendix A. 


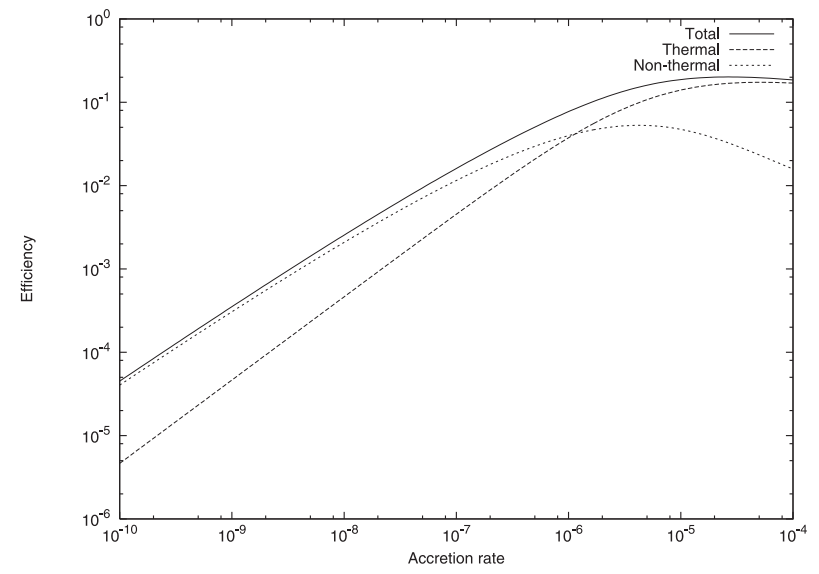

Fig. 7. Efficiencies of the synchrotron emission of thermal and nonthermal electron components of the accretion flow.

So, for the efficiency of the non-thermal component emission we have

$$
\begin{aligned}
\eta_{\mathrm{nt}}= & \frac{a_{0} \xi \dot{m}}{12} \int_{1}^{\infty} \frac{\mathrm{d} R_{0}}{R_{0}^{2}} \int_{1}^{R_{0}} \frac{\mathrm{d} R}{R^{2}} K(R) \\
& \times \mathfrak{F}_{2}\left(\left(\frac{R}{R_{0}}\right)^{5 / 8}, \frac{A \Gamma\left(R_{0}\right)}{R}\left(1-\left[\frac{R}{R_{0}}\right]^{13 / 8}\right)\right) .
\end{aligned}
$$

The results of numerical computations according to these formulae may be seen in Fig. 7.

\section{Properties of flares}

We now discuss some temporal properties of the accretion flow emission. Neglecting the complex spatial structure of the accretion flow we may consider the thermal electron component emission $L_{0}$ as a constant background with a highly variable nonthermal emission component superimposed. The variability of the latter is mostly due to the discrete nature of particle acceleration in the current sheets. Assuming for simplicity that each non-thermal electron accelerates only once and then free-falls and evolves due to adiabatic heating and radiative losses only, we get for the light curve of a single flare (i.e. a beam of $N$ electrons ejected by a reconnection event at some distance $R_{0}$ with distribution function (23) and mean gammafactor $\left.\Gamma\left(R_{0}\right)=\Delta \tau\left(R_{0}\right)\right)$ an expression

$\Delta L=\frac{4}{3} \sigma_{\mathrm{T}} c\left(\bar{\gamma}_{\mathrm{b}}^{2}-12 \tau^{2}\right) \frac{B^{2}}{8 \pi} N K(R)$

where the quantity $\bar{\gamma}_{\mathrm{b}}^{2}$ is derived in Appendix A and represents the mean square of the gamma-factor of the electron beam ejected at $R_{0}$ and free-fallen to $R$. The mean square of the Maxwellian distribution (36) gamma-factor value of $12 \tau^{2}$ is also taken into account (we need to subtract this term since each electron must occur in the total luminosity expression only once). The $K(R)$ coefficient describes relativistic effects of the radiation reduction.

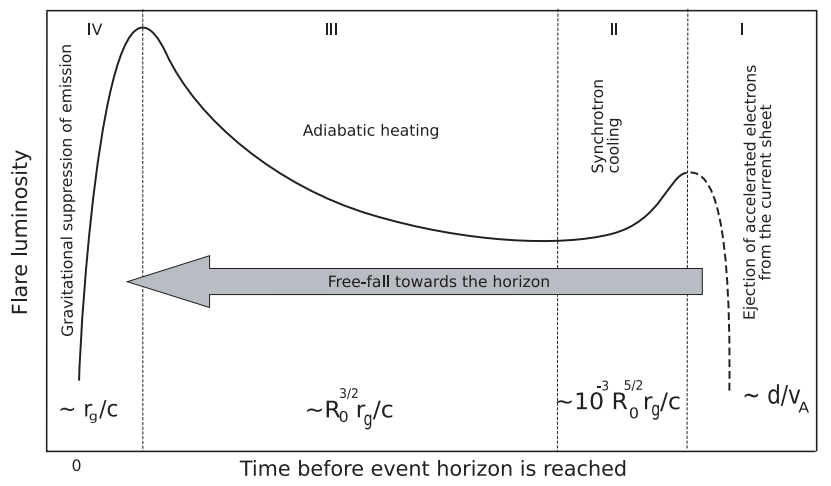

Fig. 8. Internal structure of a flare as a reflection of the electron cloud evolution. The prevailing physical mechanisms defining the observed emission are denoted and typical durations of the stages are shown.

By introducing an effective volume of the current sheet $V=N / n\left(R_{0}\right)$ and dividing by the mean luminosity of the nonthermal component $L_{\mathrm{t}}=\eta_{\mathrm{nt}} \dot{M} c^{2}$, we get

$\frac{\Delta L}{L_{\mathrm{nt}}}=\frac{\dot{m}}{12 \pi \eta_{\mathrm{nt}} R_{0}^{3 / 2}}\left(\frac{V}{r_{\mathrm{g}}^{3}}\right) \frac{\overline{\gamma_{\mathrm{b}}^{2}}-12 \tau^{2}}{R^{5 / 2}} K(R)$.

Transition to the light curve may be performed by substituting the dependence of distance on time for the falling gas element as

$R=\left(R_{0}^{3 / 2}-\frac{3 \alpha}{2} \frac{c}{r_{\mathrm{g}}} t\right)^{2 / 3}$

The temporal structure of the flare is complex (see Fig. 8). It consists of several parts with the domination of different physical processes on the electron cloud evolution. The first stage is the acceleration of the electrons in the current sheet (see Sect. 2.4), which occurs on the Alfvenic time scale, but the exact temporal structure depends on the properties of the current sheet and thus is beyond the scope of the article. The second stage is the fast emission decay due to synchrotron energy losses on the corresponding energy scale; it lasts until the establishment of the equilibrium between energy losses and adiabatic heating rates. The third stage is generally the longest one, at least for $R_{0}<10^{3}$, and has the time scale of a free-fall. Finally, near the event horizon the relativistic effects (time contraction, gravitational redshift, Doppler effect and the photons capture by the $\mathrm{BH}$ ) prevail and flare emission decays on the $r_{\mathrm{g}} / c$ time scale as the electron cloud approaches the horizon. This stage is very important as it directly reflects the metrics near the black hole.

Figure 9 shows the sample light curves of flares with $R_{0}=$ 5, $R_{0}=10, R_{0}=20$ and $R_{0}=30$, for the accretion rate $\dot{m}=$ $10^{-8}$ and the equivalent volume $V=r_{\mathrm{g}}^{3}$. The unknown first stage (flare front) is excluded.

By averaging over the flare light curve we may get the dependence of mean flare amplitude $\left\langle\Delta L / L_{\mathrm{nt}}\right\rangle$ on the ejection radius $R_{0}$. This is shown in Fig. 10. It is clear that the strongest flares are produced by reconnections very near the event 


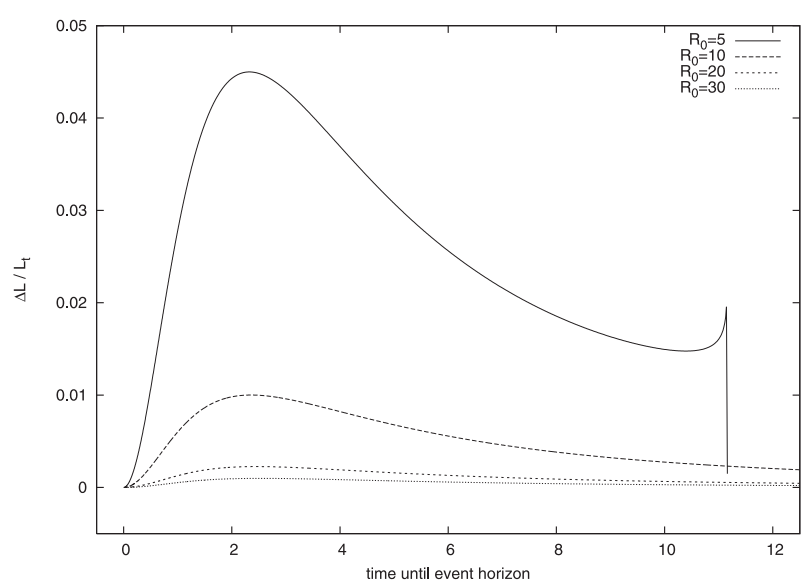

Fig. 9. Light curves of separate non-thermal flares - beams of accelerated electrons ejected at $R_{0}-$ for $\dot{m}=10^{-8}$. Time is measured in units of $r_{\mathrm{g}} / c$, luminosity in units of total thermal luminosity. The stage of particle acceleration (which defines the flare front) is omitted as its shape depends on an unknown temporal structure of the particle acceleration process, which is beyond the scope of our consideration.

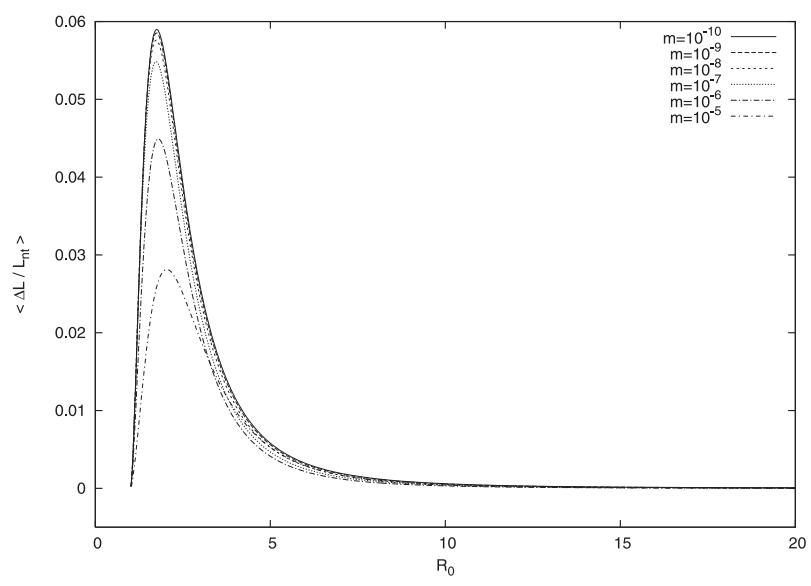

Fig. 10. Mean amplitude of a flare as a function of ejection radius $R_{0}$.

horizon (at several $r_{\mathrm{g}}$ ). The rate of a flares may be easily computed as

$$
\frac{\mathrm{d} \boldsymbol{\aleph}}{\mathrm{d} R_{0} \mathrm{~d} t}=\frac{\mathrm{d} E}{\mathrm{~d} R_{0} \mathrm{~d} t} \frac{\xi}{E \Gamma\left(R_{0}\right)} \int_{1}^{R_{0}}\left(\frac{\mathrm{d} \bar{\gamma}_{\mathrm{b}}}{\mathrm{d} R}\right)_{\mathrm{emis}} K(R) \mathrm{d} R
$$

where $E$ is the total energy of a flare as is seen at infinity

$E=L_{\mathrm{nt}} \int\left\langle\Delta L / L_{\mathrm{nt}}\right\rangle \mathrm{d} t$

By integrating this expression over the range of $R_{0}$ where $\left\langle\Delta L / L_{\mathrm{nt}}\right\rangle$ is greater than some threshold value we may get the rate of flares with given amplitudes. Results of such computation for the rate of flares with an amplitude greater than $0.1 \%$ of the mean nonthermal luminosity $\left(\left\langle\Delta L / L_{\mathrm{nt}}\right\rangle>10^{-3}\right)$ are given in the Table 1.

The fine temporal structure of these flares may be related to the motion of the electron beam in the magnetic field (this motion, as it has been noted before, is finite; electrons do not leave the small volume with the size determined by the Larmor radius and magnetic field line topology). So, if the characteristic size of the beam (the characteristic timescale of particle ejection multiplied by the speed of light) is smaller than that of the magnetic field loops, then we see the beam emission only when it is pointed towards us. The timescale of such flares is much shorter than the free-fall one, and they can be detected only in high time resolution observations. On the other hand, their properties reflect the magnetic field structure, and so its search is very important.

A significant amount of nonthermal synchrotron emission also falls in the optical range (see Table 1 and Fig. 11). This means that short flares like that shown in Fig. 9 may be detected in observations with a $1 \mu$ s time resolution at the $6 \mathrm{~m}$ telescope in the framework of the MANIA experiment (Beskin et al. 1997).

\section{Discussion}

The analysis of existing data on possible black hole masses and velocities is performed in comparison with the interstellar medium structure. It is shown that in the majority of cases in the Galaxy (>90\%), the accretion rate $\dot{m}=\dot{M} c^{2} / L_{\text {edd }}$ cannot exceed $10^{-6}-10^{-7}$ (see Fig. 1). The basis of our analysis is the assumption of energy equipartition in the accretion flow of Shvartsman (1971). Currently, there have been no successful attempts to build a magnetized plasma accretion theory without this assumption - the work of Kowalenko \& Melia (1999) led to unphysical results, while Scharlemann (1983) has some mathematical errors in computing the back-reaction of the magnetic field on the matter.

The accreting plasma is initially collisionless, and it remains so until the event horizon. The electron-electron and electron-ion free path $\lambda \sim 2.4 \times 10^{3} T^{2} n^{-1}$ even at the capture radius is as high as $\sim 10^{12} \mathrm{~cm}$. Only the magnetic fields trapped in plasma (the proton Larmor radius at $r_{\mathrm{g}}$ is $10 \mathrm{~cm}$ ) make it possible to consider the problem as a quasi-hydrodynamical one; it is only due to the magnetic field that the particle's momentum is not conserved, allowing particles to fall towards the black hole. In addition, the magnetic field effectively "traps" particles in a "box" of variable size, which allows us to consider its adiabatic heating during the fall; a correct treatment of such a process shows that for magnetized plasma such heating is $25 \%$ more effective than for ideal gas (see Sect. 3.1). Therefore, the plasma temperature in the accretion flow grows much faster and electrons become relativistic earlier $-R_{\text {rel }} \approx 6000$ in contrast to $R_{\text {rel }} \approx 1300$ in Bisnovatyi-Kogan \& Ruzmaikin (1974) and $R_{\text {rel }} \approx 200$ in Ipser \& Price (1982). The accretion flow is much hotter, and our estimation of "thermal" luminosity

$L=9.6 \times 10^{33} M_{10}^{3} n_{1}^{2}\left(V^{2}+c_{\mathrm{s}}^{2}\right)_{16}^{-3} \mathrm{erg} / \mathrm{s}$

is significantly higher than those of Ipser \& Price (1982)

$L_{\mathrm{IP}}=1.6 \times 10^{32} M_{10}^{3} n_{1}^{2}\left(V^{2}+c_{\mathrm{s}}^{2}\right)_{16}^{-3} \mathrm{erg} / \mathrm{s}$

and Bisnovatyi-Kogan \& Ruzmaikin (1974)

$L_{\mathrm{BKR}}=2 \times 10^{33} M_{10}^{3} n_{1}^{2}\left(V^{2}+c_{\mathrm{s}}^{2}\right)_{16}^{-3} \mathrm{erg} / \mathrm{s}$,

while the optical spectral shape is nearly the same. 
Table 1. Thermal and nonthermal luminosity, magnitudes, fluxes and flares rate of a $10 M_{\odot}$ black hole in a various ISM conditions at a $100 \mathrm{pc}$ distance.

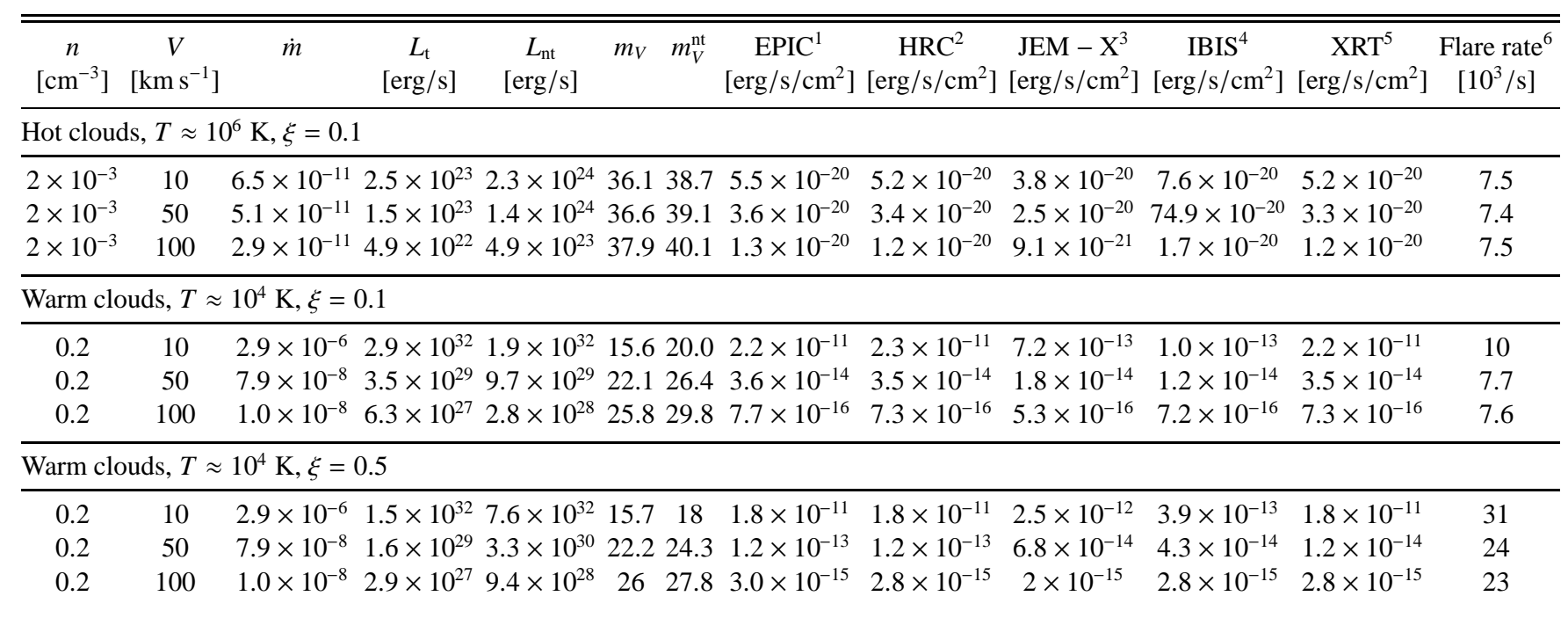

${ }^{1}$ XMM EPIC band flux. Sensitivity is $10^{-14} \mathrm{erg} / \mathrm{s} \mathrm{cm}^{2}$ for typical survey exposures.

${ }^{2}$ Chandra HRC band flux. Sensitivity ranges from $10^{-14} \mathrm{erg} / \mathrm{s} \mathrm{cm}^{2}$ to $10^{-16} \mathrm{erg} / \mathrm{s} \mathrm{cm}^{2}$ (deep surveys).

${ }^{3}$ INTEGRAL Jem-X band flux. Sensitivity is $4 \times 10^{-11} \mathrm{erg} / \mathrm{s} \mathrm{cm}^{2}$ for $100 \mathrm{ks}$ exposure.

${ }^{3}$ INTEGRAL IBIS band flux. Sensitivity is $10^{-15} \mathrm{erg} / \mathrm{s} \mathrm{cm}^{2}$ for $1000 \mathrm{ks}$ exposure.

${ }^{5}$ Swift XRT band flux. Sensitivity is $5 \times 10^{-13} \mathrm{erg} / \mathrm{s} \mathrm{cm}^{2}$ for $1 \mathrm{ks}$ exposure.

${ }^{6}$ Flare rate is for the flares with mean luminosity greater than $0.1 \%$ of mean nonthermal one.

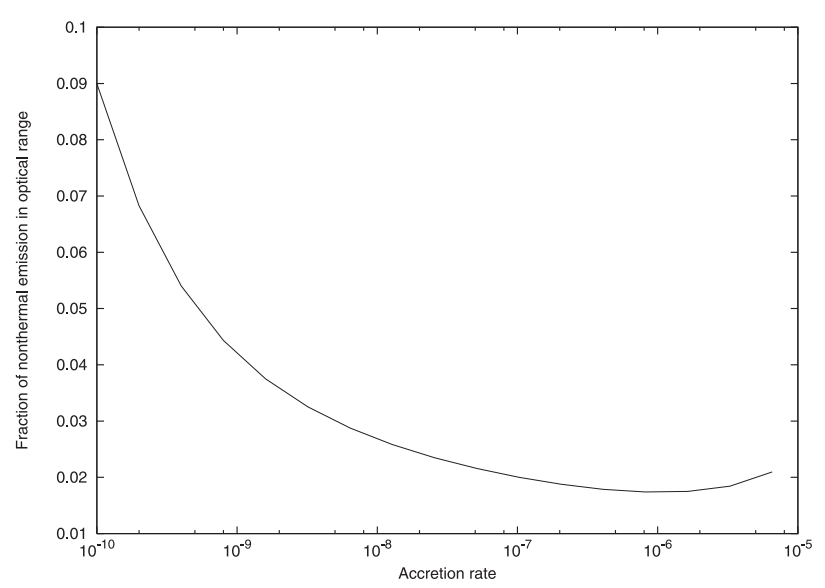

Fig. 11. Contribution of nonthermal component to the optical emission $L_{\mathrm{nt}}^{\mathrm{opt}} / L^{\mathrm{opt}}$ as a function of accretion rate.

We considered dissipation of the magnetic energy in the turbulent current sheets (Pustilnik 1997) as a mechanism supporting equipartition. In this process, the electrons are ejected with plasma from the current sheet and are accelerated. These electrons have a power-law energy distribution and its emission spectrum is flat up to the gamma band (Figs. 5 and 6). An important property of the nonthermal emission is its flaring nature - the electron ejection process is discrete; typical light curves of single beams are shown in Figs. 8 and 9.

Table 1 summarizes the observable parameters of accreting black holes.
The black holes have significant luminosity only when they are located in the warm hydrogen regions that occupy about $50 \%$ of the Galaxy volume (McKee \& Ostriker 1977). In such cases the dimensionless accretion rate lies in the $10^{-8}$ $10^{-6}$ range, and the bolometric luminosity of the accretion flow is $3 \times 10^{28}-10^{33} \mathrm{erg} / \mathrm{s}$, depending on the $\mathrm{BH}$ velocity and the fraction of magnetic energy transformed into the motion of electrons (see Table 1). As a result, the black hole at a $100 \mathrm{pc}$ distance (a sphere with this radius must contain several tens of such objects, see Beskin et al. 2000, and Agol \& Kamionkowski 2002) looks like a $15-25^{\mathrm{m}}$ optical object (due to the "thermal" spectral component) with a strongly variable companion in high-energy spectral bands ("nonthermal" component). The hard emission consists of flares, the majority of which are generated inside a $5 r_{\mathrm{g}}$ distance from the $\mathrm{BH}$ (see Figs. 8-10). These events have the durations $\sim r_{\mathrm{g}} / c\left(\sim 10^{-4} \mathrm{~s}\right)$, a rate of $10^{3}-10^{4}$ flares per second, and an amplitude of $2 \%-6 \%$. As it is seen from Table 1, the BH variable X-ray emission may be detected by modern space-borne telescopes.

Optical emission consists of both a quasistationary "thermal" part and a low-frequency tail of nonthermal flaring emission. The rate and duration of optical flares are the same as X-ray ones, while their amplitudes are significantly smaller. Indeed, the contribution of nonthermal component to the optical emission (see Fig. 11) is approximately $2 \times 10^{-2}$ for $\dot{m}=10^{-8}-10^{-6}$, so the mean amplitudes of optical flares are $0.04 \%-0.12 \%$, while the peak ones may be $1.5-2$ times higher and reach $0.2 \%$. Certainly, it is nearly impossible to detect such single flares, but their collective power reaches $18-24^{\mathrm{m}}$ (see 
Table 1) and thus may be detected in observations with high time resolution $\left(<10^{-4} \mathrm{~s}\right)$ by the large optical telescopes.

Our separation of the accretion flow emission into stationary "thermal" and flaring "nonthermal" components is a rough approximation made for the estimation of the qualitative picture of $\mathrm{BH}$ observational appearances. The real behaviour of the accreting plasma may be much more complicated.

The accretion flow is a complex dynamical system with nonlinear feedback. This is ensured by the plasma oscillations generated in each reconnection event, with beams of accelerated electrons and clouds of magnetized plasma ejected from current sheets. All these agents may act as triggers for already "prepared" inhomogeneities which turn on magnetic energy dissipation processes. This situation seems to be similar to the Solar one which determines its flaring activity, and also to the case of UV Cet stars and maybe accretion disks of X-ray binaries and active galactic nuclei. All these non-stationary processes are characterized by power-law scalings of flare energies with similar slopes of 1.5-2 at a very wide range of energies from $10^{23} \mathrm{erg} / \mathrm{s}$ for the Sun to $10^{45} \mathrm{erg} / \mathrm{s}$ for quasars. The universality of these processes may be interpreted in the framework of a fractal approach as done by Bak et al. (1987), Lu \& Hamilton (1991), Lu et al. (1993), Anastasiadis e al. (1997), Kawaguchi \& Mineshige (1999), Pustilnik (1997). This means the realization (at least in active phases) of some collective state, sometimes called "self-organized criticality", Bak et al. 1987), which is characterized by the same behaviour of the parameters on all scales. These are percolation processes. There is evidence that accretion flow is in this state, and so its observational appearance (at least that related to non-stationary processes) may be predicted and interpreted in the framework of this approach. Initial steps in this direction have been made by Beskin \& Karpov (2002), but need to be refined.

\section{Conclusions}

During recent years the number of works dealing with single stellar-mass black holes has significantly increased. Some are purely theoretical (Punsly 1998a, 1998b; Gruzinov \& Quataert 1999; Abramowicz et al. 2002) and other provide discussions of their observational detection (Heckler \& Colb 1996; Fujita et al. 1998; Beskin et al. 2000; Agol \& Kamionkowski 2002; Chisholm et al. 2003). The importance of experiments in strong gravitational fields has been recently noted by Damour (1998). Kramer et al. (2004) discussed the new possibilities of black holes metric study by the investigation of radio pulsar - $\mathrm{BH}$ binary systems with new generation of radio telescopes.

In this work we tried to concretize physical properties of plasma accreted onto the black hole within the classical paradigm of equipartition of Shvartsman (1971). Assuming the discrete nature of the magnetic energy dissipation processes in current sheets allows us to clarify the shape of the synchrotron spectrum of the accretion flow. A hard highly non-stationary nonthermal spectral component appears as an emission of accelerated particles. The beams accelerated in the current sheets can generate very short flares, providing information about the neighborhood of the event horizon (Fig. 9). On the other hand it is clear from Fig. 12 that at low accretion rates a significant

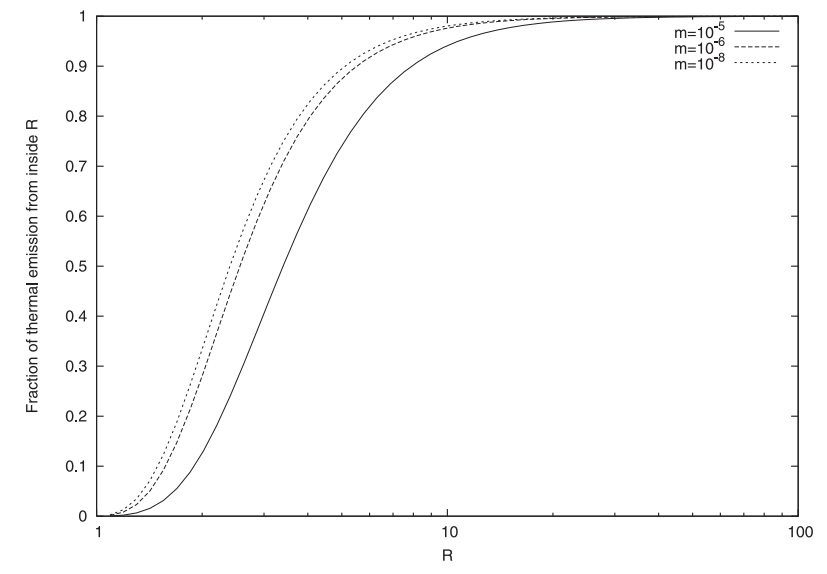

Fig. 12. Fraction of thermal synchrotron emission that comes from inside a given radius $R$.

amount of thermal synchrotron radiation is generated inside $3 r_{\mathrm{g}}$ - this means that the behaviour of this component will reflect the properties of space-time in strong gravitational fields too.

It is clear that the search for a black hole strategy may be modified in accordance with such results. Optical high time resolution studies of X-ray sources may be very important. Single black holes may be contained inside the known stationary gamma sources (Gehrels et al. 2000) as well as objects causing long microlensing events (Paczynski 1991). Thus it is very important to look for X-ray emission as well as for fast optical variability of these objects. Sample observations of the longest microlensing event MACHO 1999-BLG-22 (Bennett et al. 2002), a stellar-mass black hole candidate, have been performed at the Special Astrophysical Observatory of RAS in the framework of the MANIA experiment in 2003-2004 (Beskin et al. 2005).

The best evidence will be provided by the synchronous high time resolution observations in optical and X-ray ranges.

Detection of the event horizon signatures cannot result from statistical studies. A detailed study of each object is needed to detect its specific appearance.

Acknowledgements. This work has been supported by the Russian Foundation for Basic Research (grants Nos. 01-02-17857 and 0402-17555) and by the grant in the framework of the CNR (Italy) RAS (Russia) agreement on scientific collaboration. We also thank T.Tupolova for help in manuscript preparation. S.K. thanks the Russian Science Support Foundation for support. G.B. thanks the Cariplo Foundation for the scholarship and Merate Observatory for hospitality. The Authors thank Dr. L.Pustilnik for exciting discussions. The authors thank the anonymous referee for the valuable comments.

\section{Appendix A: Moments of single accelerated beam energy distribution}

For the case of the electron beam ejected from the current sheet at some radius $R_{0}$ and free-falling to $R$ not interacting with the 
background particles and beams ejected below the moments of the energy distribution may be written using (46) as

$\bar{\gamma}_{\mathrm{b}}(R)=\left(\int_{1}^{\infty} f\left(R_{0}, \gamma_{0}\right) \mathrm{d} \gamma_{0}\right)^{-1} \int_{1}^{\infty} \frac{f\left(R_{0}, \gamma_{0}\right) \gamma_{0} \mathrm{~d} \gamma_{0}}{C_{1}\left(R, R_{0}\right) \gamma_{0}+C_{2}\left(R, R_{0}\right)}(\mathrm{A} .1)$

$\bar{\gamma}_{\mathrm{b}}^{2}(R)=\left(\int_{1}^{\infty} f\left(R_{0}, \gamma_{0}\right) \mathrm{d} \gamma_{0}\right)^{-1} \int_{1}^{\infty} \frac{f\left(R_{0}, \gamma_{0}\right) \gamma_{0}^{2} \mathrm{~d} \gamma_{0}}{C_{1}\left(R, R_{0}\right) \gamma_{0}+C_{2}\left(R, R_{0}\right)}$

For the initial distribution of the form (23) this may be evaluated as

$\bar{\gamma}_{\mathrm{b}}(R)=\Gamma\left(R_{0}\right) \mathfrak{\mho}_{1}\left(\sqrt{\frac{R}{R_{0}}}, \frac{A \Gamma\left(R_{0}\right)}{R}\left(1-\left[\frac{R}{R_{0}}\right]^{3 / 2}\right)\right)$

$\bar{\gamma}_{\mathrm{b}}^{2}(R)=\Gamma^{2}\left(R_{0}\right) \mathfrak{\mho}_{2}\left(\sqrt{\frac{R}{R_{0}}}, \frac{A \Gamma\left(R_{0}\right)}{R}\left(1-\left[\frac{R}{R_{0}}\right]^{3 / 2}\right)\right)$

where new functions

$$
\begin{aligned}
\mathfrak{F}_{1}(A, B) & =\int_{0}^{\infty} \frac{x \mathrm{e}^{-x} \mathrm{~d} x}{A x+B} \\
& =\frac{1}{A^{2}}\left(A-B \cdot \exp \left(\frac{B}{A}\right) E \mathrm{i}_{1}\left(\frac{B}{A}\right)\right) \\
\mathfrak{F}_{2}(A, B) & =\int_{0}^{\infty} \frac{x \mathrm{e}^{-x} \mathrm{~d} x}{(A x+B)^{2}} \\
& =\frac{1}{A^{3}}\left(-A+(1+B) \cdot \exp \left(\frac{B}{A}\right) E \mathrm{i}_{1}\left(\frac{B}{A}\right)\right)
\end{aligned}
$$

are introduced and an expression for the integral exponent

$E \mathrm{i}_{n}(x)=\int_{1}^{\infty} \frac{\mathrm{e}^{-\xi x} \mathrm{~d} \xi}{\xi^{n}}$

is used.

\section{References}

Abramowicz, M. A., Kluzniak, W., \& Lasota, J. P. 2002, A\&A, 396, 31

Agol, E., \& Kamionkowski, M. 2002, MNRAS, 334, 553

Anastasiadis, A., Vlahos, L., \& Georgoulis, M. K. 1997, ApJ, 489, 367

Armstrong, J. W., Rickett, B. J., Spangler, S. R. 1995, ApJ, 443, 209

Bak, P., Tang, C., \& Weisenfeld, K. 1987, Phys. Rev. Lett., 59, 381

Bennett, D. P., Becker, A. C., Calitz, C. C., et al. 2002, ApJ, 579, 639

Beskin, G. M., \& Shvartsman, V. F. 1976, in Relativistic Astrophysics, Cosmology and Gravitational Experiment, 9

Beskin, G. M., \& Mitronova, S. N. 1991, Bull. SAO RAS, 32, 33

Beskin, G. M., Komarova, V. N., Neizvestny, S. I., et al. 1997, ExA, 7, 413

Beskin, G.M., Shearer, A., Golden, A., et al. 2000, in Texas in Paris, CD-ROM 12/06

Beskin, G. M., \& Karpov, S. V. 2002a, Gravit. Cosmol. Suppl. Ser., 8, 182

Beskin, G. M., \& Tuntsov, A. V. 2002b, A\&A, 394, 489
Beskin, G. M., Karpov, G. M., Plokhotnichenko, V. L., et al. 2005, in preparation

Birk, G. T., Lesch, H., Schopper, R., \& Wiechen, H. 1999, APh, 11, 63

Bisnovatyi-Kogan, G. S., \& Ruzmaikin, A. A. 1974, Ap\&SS, 28, 45

Bisnovatyi-Kogan, G. S., \& Lovelace, R. V. E. 1997, ApJ, 486, L43

Bisnovatyi-Kogan, G. S., \& Lovelace, R. V. E. 2000, ApJ, 529, 978

Bisnovatyi-Kogan, G. S., \& Lovelace, R. V. E. 2001, NewAR, 45, 663

Bondi, H., \& Hoyle, F. 1944, MNRAS, 104, 273

Bondi, H. 1952, MNRAS, 112, 195

Chakrabarti, S. K. 1996, Phys. Rep., 266, 229

Cherepashchuk, A. M., Usp. Fiz. Nauk, 173, 345

Chisholm, J. R., Dodelson, S., \& Kolb, E. W. 2003, ApJ, 596, 437

Coppi, P. S. 1999, in High Energy Processes in Accreting Black Holes, ASP Conf. Ser., 161, ed. J. Poutanen, \& R. Svensson

Damour, T. 1998, 19th Texas Symposium on Relativistic Astrophysics and Cosmology Proceedings, 371

Davies, R. E., \& Pringle, J. E. 1980, MNRAS, 191, 599

Dere, K. P. 1996, ApJ, 472, 864

Falgarone, E., \& Phillips, T. G. 1990, ApJ, 359, 344

Font, J. A., \& Ibanez, J. M. 1998, ApJ, 494, 297

Fryer, C. L., \& Kalogera, V. 2001, ApJ, 554, 548

Fujita, Y., Inoue, S., Nakamura, T., et al. 1998, ApJ, 495, 85

Gehrels, N., Macomb, D. J., Bertsch, D. L., et al. 2000, Nature, 404, 363

Gershberg, R. E. 1989, Mem. Soc. Ast. Ital., 59, 1

Greiner, J., Cuby, J.-G., \& Mc Caughrean, M. J. 2001, Nature, 414, 522

Gruzinov, A., \& Quataert, E. 1999, ApJ, 520, 248

Heckler, A. F., \& Colb, E. W. 1996, ApJ, 472, 85

Hudson, Y., \& Ryan, J. 1995, ARA\&A, 33, 239

Illarionov, A. F., \& Sunyaev, R. A. 1975, A\&A, 39, 185

Innes, D. E., Inhester, B., Axford, W. L., \& Wilhelm, K. 1997, Nature, 386,811

Ipser, J. R., \& Price, R. H. 1977, ApJ, 216, 578

Ipser, J. R., \& Price, R. H. 1982, ApJ, 255, 654

Kaplan, S. A, \& Tsytovich, V. N. 1973, Plasma Astrophysics, International Series of Monographs in Natural Philosophy (Oxford: Pergamon Press)

Kawaguchi, T., \& Mineshige, S. 1999, PASP, 52, L1

Kowalenko, V., \& Melia, F. 1999, MNRAS, 310, 1053

Kramer, M., Backer, D. C., Cordes, J. M., et al, 2004, NewAR, 48, 993

Landau, L. D., \& Lifshitz, E. M. 1971, Classical theory of fields, Course of theoretical physics - Pergamon International Library of Science, Technology, Engineering and Social Studies (Oxford: Pergamon Press)

Larson, R. B. 1981, MNRAS, 194, 809

Lazarian, A., \& Vishniac, E. T. 1999, ApJ, 517, 700

Lazarian, A., \& Vishniac, E. T. 2001, in Astrophysical Plasmas: Codes, Models \& Observations, ed. S. J. Arthur, N. Brickhouse, \& J. Franco

Lightman, A. P., \& Rybicki, G. B. 1979, Rad. Proc. Astrophys.

Lu, E. T., \& Hamilton, R. J. 1991, ApJ, 380, 89

Lu, E.T., Hamilton, R.J., McTiernan, \& J. M. Bromund, K. 1993, ApJ, 412,841

Mahadevan, R., \& Quataert, E. 1997, ApJ, 490, 605

McKee, C. F., \& Ostriker, J. P 1977, ApJ, 218, 148

Meszaros, P. 1975, A\&A, 44, 59

Miller, J. S., Shahbaz, T., \& Nolan, L. A. 1998, MNRAS, 294, 25

Miller, J. M., Raymond, J., Fabian, A. C. et al. 2004, ApJ, 601, 450

Miniutti, G., Fabian, A. C., \& Miller, J. M. 2004, MNRAS, 351, 466

Oppenheimer, J., \& Snyder, H. 1939, Phys. Rev., 56, 455 
Paczynski, B. 1991, ApJ, 371, 63

Parker, E. N. 1979, Cosmic Magnetic Fields (Oxford: Clarendon press)

Petchek, H. E. 1963, AAS-NASA Symp. Phys. Solar Flares, 426

Punsly, B. 1998a, ApJ, 498, 640

Punsly, B. 1998b, ApJ, 498, 660

Pustilnik, L. A. 1978, Sov. Ast., 22, 350

Pustilnik, S. A. 1977, Soobsch. SAO, 18, 3

Pustilnik, L. A. 1997, Ap\&SS, 252, 325

Reynolds, ó. S., \& Nowak, M. A. 2003, PhR, 377, 389

Reynolds, C. S., Brenneman, L. W., Garofalo, D., et al. 2004, in From X-ray Binaries to Quasars: Black hole Accretion on All Mass Scales, ed. T. Maccarone, R. Fender, \& L. Ho, in press

Roberts, T. P., Goad, M. R., Ward, M. J., et al. 2002, Proc. of the Symposium, New Visions of the X-ray Universe in the XMM-Newton and Chandra Era, 26-30 November 2001

Ruffert, M. 1997, A\&A, 317, 793

Ruffert, M. 1999, A\&A, 346, 861
Sawada, K., Matsuda, T., Anzer, U., et al. 1989, A\&A, 221, 263

Shapiro, S. L. 1973a, ApJ, 180, 531

Shapiro, S. L. 1973b, ApJ, 185, 69

Shapiro, S. L. 1974, ApJ, 189, 343

Scharlemann, E. T. 1983, ApJ, 272, 279

Shakura, N. I., \& Sunyaev, R. A. 1973, A\&A, 24, 337

Shields, G. A. 1999, PASP, 111, 661

Shvartsman, V. F. 1971, AZh, 48, 438

Shvartsman, V. F. 1977, Soobsch. SAO, 19, 3

Shvartsman, V. F., Beskin, G. M., \& Pustilnik, S. A. 1989a, Afz, 31, 457

Shvartsman, V. F., Beskin, G. M., \& Mitronova, S. N. 1989b, Astron. Rep. Lett., 15, 145

Spitzer, L. 1954, ApJ, 120, 1

Sweet, P. A. 1969, ARA\&A, 7, 149

Syrovatskii, S. I. 1981, ARA\&A, 19, 163

Will, C. M. 1998 [gr-qc/9811036] 